\title{
SN 2019hcc: a Type II supernova displaying early O II lines
}

\author{
Eleonora Parrag ${ }^{\circledR},{ }^{1 \star}$ Cosimo Inserra ${ }^{\circledR},{ }_{1}$ Steve Schulze ${ }^{\circledR}, 2$ Joseph Anderson, ${ }^{3}$ Ting-Wan Chen, ${ }^{2,4}$
} Giorgios Leloudas, ${ }^{5}$ Lluis Galbany ${ }^{\circledR}, 6$ Claudia P. Gutiérrez, ${ }^{7,8}$ Daichi Hiramatsu, ${ }^{9,10}$ Erkki Kankare, ${ }^{8}$ Tomás E. Müller-Bravo, ${ }^{11}$ Matt Nicholl, ${ }^{12}$ Giuliano Pignata, ${ }^{13,14}$ Regis Cartier, ${ }^{15}$ Mariusz Gromadzki ${ }^{\circledR}, 16$ Alexandra Kozyreva ${ }^{\circledR},{ }^{17}$ Arne Rau, ${ }^{4}$ Jamison Burke,,${ }^{9,10}$ D. Andrew Howell, ${ }^{9,10}$ Curtis McCully ${ }^{9}$ and Craig Pellegrino 9,10

${ }^{1}$ School of Physics \& Astronomy, Cardiff University, Queens Buildings, The Parade, Cardiff CF24 3AA, UK

${ }^{2}$ The Oskar Klein Centre, Department of Astronomy, Stockholm University, AlbaNova, SE-10691 Stockholm, Sweden

${ }^{3}$ European Southern Observatory, Alonso de Cordova 3107, Casilla 19, 19001 Santiago, Chile

${ }^{4}$ Max-Planck-Institut für Extraterrestrische Physik, Giessenbachstraße 1, D-85748 Garching, Germany

${ }^{5}$ DTU Space, National Space Institute, Technical University of Denmark, Elektrovej 327, DK-2800 Kgs. Lyngby, Denmark

${ }^{6}$ Departamento de Física Teórica y del Cosmos, Universidad de Granada, E-18071 Granada, Spain

${ }^{7}$ Finnish Centre for Astronomy with ESO (FINCA), University of Turku, FI-20014 Turku, Finland

${ }^{8}$ Tuorla Observatory, Department of Physics and Astronomy, University of Turku, FI-20014 Turku, Finland

${ }^{9}$ Las Cumbres Observatory, 6740 Cortona Drive, Suite 102, Goleta, CA 93117-5575, USA

${ }^{10}$ Department of Physics, University of California, Santa Barbara, CA 93106-9530, USA

${ }^{11}$ School of Physics and Astronomy, University of Southampton, Southampton, Hampshire SO17 1BJ, UK

${ }^{12}$ Birmingham Institute for Gravitational Wave Astronomy and School of Physics and Astronomy, University of Birmingham, Birmingham B15 2TT, UK

${ }^{13}$ Departamento de Ciencias Fisicas, Universidad Andres Bello, Avda. Republica 252, 8320000 Santiago, Chile

${ }^{14}$ Millennium Institute of Astrophysics (MAS), Nuncio Monseñor Sotero Sanz 100, Providencia, Santiago, Chile

${ }^{15}$ Cerro Tololo Inter-American Observatory, NSF's National Optical-Infrared Astronomy Research Laboratory, Casilla 603, La Serena, Chile

${ }^{16}$ Astronomical Observatory, University of Warsaw, Al. Ujazdowskie 4, Pl-00-478 Warszawa, Poland

${ }^{17}$ Max-Planck-Institut für Astrophysik, Karl-Schwarzschild-Str. 1, D-85748, Garching, Germany

Accepted 2021 July 15. Received 2021 July 14; in original form 2021 April 30

\section{ABSTRACT}

We present optical spectroscopy together with ultraviolet, optical, and near-infrared photometry of SN 2019hcc, which resides in a host galaxy at redshift 0.044 , displaying a sub-solar metallicity. The supernova spectrum near peak epoch shows a ' $w$ ' shape at around $4000 \AA$ which is usually associated with O II lines and is typical of Type I superluminous supernovae. SN 2019hcc post-peak spectra show a well-developed $\mathrm{H} \alpha$ P-Cygni profile from $19 \mathrm{~d}$ past maximum and its light curve, in terms of its absolute peak luminosity and evolution, resembles that of a fast-declining Hydrogen-rich supernova (SN IIL). The object does not show any unambiguous sign of interaction as there is no evidence of narrow lines in the spectra or undulations in the light curve. Our TARDIS spectral modelling of the first spectrum shows that carbon, nitrogen, and oxygen (CNO) at $19000 \mathrm{~K}$ reproduce the ' $\mathrm{w}$ ' shape and suggests that a combination of non-thermally excited CNO and metal lines at $8000 \mathrm{~K}$ could reproduce the feature seen at $4000 \AA$. The Bolometric light-curve modelling reveals that SN 2019 hcc could be fit with a magnetar model, showing a relatively strong magnetic field $\left(B>3 \times 10^{14} \mathrm{G}\right)$, which matches the peak luminosity and rise time without powering up the light curve to superluminous luminosities. The high-energy photons produced by the magnetar would then be responsible for the detected O II lines. As a consequence, SN 2019 hcc shows that a ' $\mathrm{w}$ ' shape profile at around $4000 \AA$, usually attributed to $\mathrm{O}$ II, is not only shown in superluminous supernovae and hence it should not be treated as the sole evidence of the belonging to such a supernova type.

Key words: line: formation-line: identification-stars: magnetars.

\section{INTRODUCTION}

Historically, supernovae ( $\mathrm{SNe}$ ) were initially classified according to specific observational characteristics, and then a physically motivated classification scheme was built, providing insight into explosion physics and stellar evolution pathways. SNe can be broadly classified

^E-mail: parrage@ cardiff.ac.uk into two main types - those which show hydrogen lines (Type II) and those which do not (Type I). Core-collapse of a massive star with a retained hydrogen envelope produces the hydrogen-rich Type II SNe, whereas if such envelope has been stripped we observe stripped envelope supernovae (SESNe), which fall into the hydrogen-poor Type I.

$\mathrm{SNe}$ II are considered a single population (Minkowski 1941) but a large spectral and photometric diversity is nowadays observed (e.g. Gutiérrez et al. 2017a). SNe II were historically split into two categories based on their photometric evolution, SNe IIL showing a linear 
decline in the light curve (Barbon, Ciatti \& Rosino 1979) and SNe IIP showing a plateau for several weeks. Arcavi (2017) suggested that the difference in Type IIL, a typically brighter subclass of Type II supernovae, could be due to the presence of a magnetar. However, Anderson et al. (2014b) suggested that the diversity observed in SN II light curves and their spectra is due to the mass and density profile of the retained hydrogen envelopes. For years, it has been a matter of dispute whether IIL and IIP are a continuous population or have distinctly different physics and progenitors but, recently, increasing evidence has suggested that they are coming from a continuous populations (e.g. Anderson et al. 2014b; Sanders et al. 2015; Galbany et al. 2016; Valenti et al. 2016; de Jaeger et al. 2018). Anderson et al. (2014b) also noted that very few SNe II actually fit the classical description of SNe IIL as most show a plateau of some form. However, Davis et al. (2019) performed a spectroscopic analysis in the near-infrared (NIR) which found distinct populations corresponding to fast (SN IIL) and slow (SN IIP) decliners, though they suggested this could alternatively be accounted for by a gap in the data set.

Further splittings of SNe II are based on spectroscopic features. $\mathrm{SNe}$ IIb are transitional events between hydrogen-rich SNe II and hydrogen-poor SNe Ib (e.g. Filippenko, Matheson \& Ho 1993). SNe IIn display narrow emission lines attributed to interaction with dense circumstellar material (e.g. Schlegel 1990). SN classification can be time dependent, as some objects have been observed to dramatically change their observables over time, ranging on timescales from weeks to years. In recent years, wide-field surveys have revealed a large diversity of unusual transients that include extreme transitional objects (Modjaz, Gutiérrez \& Arcavi 2019). One such example is SN 2017ens (Chen et al. 2018), a transition between a luminous broadline SN Ic and a SN IIn. SN 2017ivv is another, sharing properties with fast-declining SN II and SN IIb (Gutiérrez et al. 2020), or SN 2014C, which underwent a change from a SN Ib to SN IIn due to interaction with a hydrogen-rich CSM (Milisavljevic et al. 2015). Objects such as these can support physical continuity between progenitors and explosion mechanisms of different types (Filippenko 1988).

Another finding of the wide-field survey has been the discovery of a population of ultra-bright 'superluminous' supernovae (Quimby et al. 2011). SLSNe are intrinsically rare with respect to common core-collapse SNe (Quimby et al. 2013; McCrum et al. 2015; Inserra 2019), with a recent measurement by Frohmaier et al. (2021) reporting a local ratio of SLSNe I to all types of CCSNe of $\sim 1 / 3500_{-720}^{+2800}$. SLSNe are characterized by absolute luminosities at maximum light of approximately -21 mag (Gal-Yam 2012; Inserra 2019), though recent evidence suggests that SLSNe in fact occupy a wider range of luminosities, with peak luminosities reportedly as faint as -20 mag (e.g. Angus et al. 2019). They are typically found in dwarf, metalpoor, and star-forming galaxies, suggesting that SLSNe are more effectively formed in low metallicity environments (e.g. Lunnan et al. 2014; Leloudas et al. 2015a; Chen et al. 2017c; Schulze et al. 2018). Type I superluminous supernovae (SLSNe I) display a lack of H or He features, and early-time spectra show prominent broad absorption features around 4200 and $4400 \AA$. These are usually associated with $\mathrm{O}$ II, consisting of a complex blend of many individual lines (Quimby et al. 2011; Gal-Yam 2019).

Here, we present the data and analysis of SN 2019hcc, which appears to show typical features of both SLSNe I and SN II at different stages in its evolution. The first spectrum appeared to contain a 'w' shape associated with O II lines near maximum, typical of SLSNe I (e.g. Quimby et al. 2011; Inserra 2019). However, subsequent spectra identify SN $2019 \mathrm{hcc}$ as a moderately bright Type II supernova, similar to those discussed in Inserra et al. (2013a), due to the presence of Balmer lines. This is the first such object (to our knowledge) to be identified in the literature.

In this paper, we will show that SN 2019hcc, despite displaying a ' $w$ ' shape profile similar to those observed in SLSNe I, otherwise conforms with the typical properties of $\mathrm{SNe}$ II. We will then investigate possible mechanisms which could be responsible for producing such a 'w' shape profile in a SN II. This paper is organized as follows. In Section 2, we report the observations and how the data were obtained and reduced. In Section 3, the host galaxy and its properties are analysed. In Section 4, the rise time and explosion epoch are determined, and the photometry is presented. Section 5 contains a detailed analysis of the optical, NIR, and bolometric lightcurve properties. Section 6 focuses on the spectra of SN 2019hcc, their comparison with other SN types which share common features, and on a close analysis of the Balmer profiles to look for signatures of interaction. Section 7 considers the ' $w$ ' profile, investigating the required conditions for the formation of the features, and discusses the merit of different powering mechanisms. Section 8 provides a summary of our work.

\section{OBSERVATIONS AND DATA REDUCTION}

SN 2019hcc was discovered by the Gaia satellite (Gaia Collaboration 2016) as Gaia19cdu on MJD 58640 (Delgado et al. 2019), and subsequently by the Asteroid Terrestrial-impact Last Alert System (ATLAS; Tonry et al. 2018; Smith et al. 2020) on MJD 58643 as ATLAS19mgw (Frohmaier et al. 2019). The first spectrum was taken on MJD 58643, $3 \mathrm{~d}$ after discovery and $7 \mathrm{~d}$ after the photometric maximum, see Section 5. It was then classified on MJD 58643 as a SLSN I (Swann et al. 2019) as a consequence of the w-shaped absorption feature around $4000 \AA$. The redshift was found to be $z=$ 0.044 from the host galaxy emission lines as visible from the second spectrum, and then confirmed by the host galaxy spectrum taken at the end of the SN campaign. We assume a flat $\Lambda \mathrm{CDM}$ universe with a Hubble constant of $H_{0}=70 \mathrm{~km} \mathrm{~s}^{-1} \mathrm{Mpc}^{-1}$ and $\Omega_{\mathrm{m}}=0.3$ and hence a luminosity distance of $194.8 \mathrm{Mpc}$.

However, the second spectrum taken on MJD 58655 showed a prominent $\mathrm{H} \alpha$ profile implying the target was not a SLSN I, but rather a bright Type II. It had equatorial coordinates of RA: 21:00:20.930, Dec.: -21:20:36.06, with the most likely host J210020.73-212037.2 in the WISEA catalogue at $M_{r}=19.3 \mathrm{mag}$ (Cutri et al. 2013), since the redshift of this host and that of SN 2019hcc are matched. The Milky Way extinction was taken from the all-sky Galactic dustextinction survey (Schlafly \& Finkbeiner 2011) as Av $=0.19$. Taking $\mathrm{Rv}=3.1$, this gives an $E(B-V)$ of 0.06 . Since there are no Na I D absorption lines related to the host and the SN luminosity and colour evolution appear to be as expected in a SN II (see Section 5), the host galaxy reddening has been assumed negligible. Fig. 1 shows the finder chart and the local environment of SN 2019hcc.

\subsection{Data reduction}

Five optical spectra were taken over a range of 5 months with the NTT+EFOSC2 at the La Silla Observatory, Chile. This was under the advanced Public ESO Spectroscopic Survey of Transient Objects programme (ePESSTO+; Smartt et al. 2015). This was alongside a host galaxy spectrum taken over a year after explosion when SN 2019hcc was no longer visible. The spectra were reduced using the PESSTO NTT pipeline. ${ }^{1}$ There was also one spectrum taken by

\footnotetext{
${ }^{1}$ https://github.com/svalenti/pessto
} 


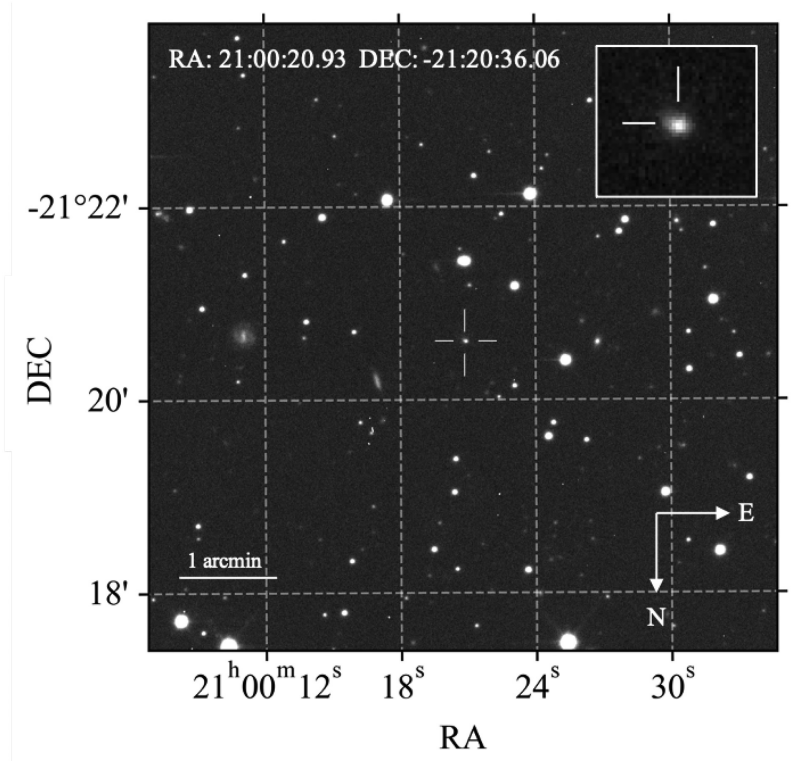

Figure 1. The finder chart for SN 2019hcc displaying the local environment, taken in $r$-band at MJD $=58660$ by LCO. The host is a low luminosity galaxy. SN $2019 \mathrm{hcc}$ is marked by the white crosshairs, and in the blow-up image in the top-right corner.

the Goodman High Throughput Spectrograph at the Southern Astrophysical Research telescope (SOAR) (Clemens, Crain \& Anderson 2004), reduced using the dedicated pipeline (Sánchez-Sáez et al. 2019). The final reduced and calibrated spectra will be available on the Weizmann Interactive Supernova Data Repository (WISeREP; Gal-Yam \& Yaron 2012).

Photometric data were obtained by the Las Cumbres Observatory (LCO; Brown et al. 2011) with the camera Sinistro built for the $1 \mathrm{~m}$-class LCO telescopes, and by the Liverpool Telescope (LT; Steele et al. 2004) on the Canary Islands. Images were combined using $\mathrm{SNOoPY}^{2}$ and the magnitudes were retrieved using PSF photometry, with the zero-point calibration completed using reference stars accessed from the Panoramic Survey Telescope and Rapid Response System (Pan-STARRS; Chambers et al. 2016) and the Vizier catalogues (Ochsenbein, Bauer \& Marcout 2000). This was performed using the code described in detail in Appendix A. Additional photometry was also taken by ATLAS, Swift + Ultraviolet/Optical Telescope (UVOT; Roming et al. 2005), and the GammaRay Burst Optical and Near-Infrared Detector (GROND; Greiner et al. 2008). GROND is an imaging instrument to investigate GammaRay Burst Afterglows and other transients simultaneously in seven bands grizJHK mounted at the 2.2-m MPG telescope at the ESO La Silla Observatory (Chile). The GROND images of SN 2019hcc were taken under the GREAT survey (Chen et al. 2018). GROND (Krühler et al. 2008), ATLAS, and Swift data were reduced using their own pipelines. The photometry and spectroscopy logs, including dates, configurations, and magnitudes are reported in Appendix B. As Swift observes simultaneously with UVOT and the X-ray Telescope (XRT), we report that the corresponding upper limit on the unabsorbed $0.3-$ $10 \mathrm{keV}$ flux is $2.6 \times 10^{-14} \mathrm{cgs}$ (assuming a power law with photon index 2 and the Galactic column density of $4.9 \times 10^{20} \mathrm{~cm}^{-2}$ ) resulting

\footnotetext{
${ }^{2}$ SNOoPy is a package for SN photometry using PSF fitting and/or template subtraction developed by E. Cappellaro. A package description can be found at http://sngroup.oapd.inaf.it/snoopy.html
}

in an upper limit on luminosity of $\sim 10^{41} \mathrm{erg} \mathrm{s}^{-1}$ at SN 2019hcc distance. The closest non-detections were taken by ATLAS from 34 to $22 \mathrm{~d}$ before discovery, with a confidence of $3 \sigma$.

\section{HOST GALAXY}

The host galaxy spectrum for SN 2019hcc was taken with NTT+EFOSC2 (Buzzoni et al. 1984) at the La Silla Observatory, Chile, on MJD 59149, when the SN was no longer visible, as part of the ePESSTO+ programme (Smartt et al. 2015). The line fluxes were measured using the splot function in IRAF (Tody 1986) by taking a number of measurements and averaging to account for the uncertainty in the location of the continuum. The host galaxy spectrum was analysed using pyMCZ. This is an open-source PYTHON code which determines the metallicity indicator, oxygen abundance $(12+\log (\mathrm{O} / \mathrm{H}))$, through Monte Carlo sampling, and gives a statistical confidence region (Bianco et al. 2016). The input of this code is the line flux and associated uncertainties for lines such as $[\mathrm{OII}]$ and $\mathrm{H} \alpha$ from the host galaxy spectrum. Kewley \& Ellison (2008) found that the choice of metallicity calibration has a significant effect on the shape and $y$-intercept $(12+\log (\mathrm{O} / \mathrm{H}))$ of the mass-metallicity relation, therefore multiple markers are used to measure the metallicity in an effort to give a representative range.

Fig. 2 shows the input (upper panel) and output (lower panel) for pyMCZ (see Bianco et al. 2016). The metallicity estimators are those of Zaritsky, Kennicutt \& Huchra (1994) [Z94], McGaugh (1991) [M91], Maiolino et al. (2008) [M08], and Kewley \& Ellison (2008) [KK04]. These metallicity markers are all based on $R_{23}$, see Bianco et al. (2016) for a summary and further details:

$R_{23}=\frac{[\mathrm{O} \text { II }] \lambda 3727+[\mathrm{O} \mathrm{III}] \lambda \lambda 4959,5007}{\mathrm{H} \beta}$

[N II] $\lambda 6584$ is not visible in this spectrum, and at this resolution it would be very difficult to resolve as it is so close to $\mathrm{H} \alpha$. A lack of $[\mathrm{N} \mathrm{II]}$ is an indicator of low metallicity, therefore the lower branches of the metallicity indicators were used in the code, apart from Z94, where only the upper branch is available in pyMCZ. The metallicity markers used are those available given the line fluxes which were input into pyMCZ, which are labelled in the top panel of Fig. 2. Averaging them we obtain a host galaxy metallicity of $12+\log (\mathrm{O} / \mathrm{H})=8.08 \pm 0.05$, which is below solar abundance.

We also note that the $\mathrm{H} \alpha / \mathrm{H} \beta$ flux ratio in the host spectrum is measured to be $2.2 \pm 0.1$, less than the intrinsic ratio 2.85 for case B recombination at $T=10^{4} \mathrm{~K}$ and $\mathrm{n}_{\mathrm{e}} \sim 10^{2}-10^{4} \mathrm{~cm}^{-3}$ (Osterbrock \& Ferland 2006). A ratio of less than 2.85 can result from an intrinsically low reddening combined with errors in the stellar absorption correction and/or errors in the line flux calibration and measurement (Kewley \& Ellison 2008).

Models by Dessart et al. (2014) hint to a lack of SNe II below $0.4 \mathrm{Z}_{\odot}$. However, this may be biased as higher luminosity hosts were used which tend to have higher metallicity. On the other hand, SLSNe I are predominantly found in dwarf galaxies, indicating that their progenitors have a low metallicity. A $0.5 \mathrm{Z}_{\odot}$ threshold has been suggested for the formation of SLSNe I (Chen et al. 2017c). Lunnan et al. (2014) found a median metallicity of $8.35=0.45 \mathrm{Z}_{\odot}$ for a sample of 31 SLSNe I.

The measured metallicity was compared to both Type II and SLSN I hosts. Table 1 contains the mean metallicity excluding Z94 (this is likely incorrect as it is the upper branch) from Fig. 2, compared to averages for SLSNe I and SNe II. Schulze et al. (2020) performed a comprehensive analysis of $\mathrm{SN}$ hosts based on a sample of $888 \mathrm{SNe}$ of 12 distinct classes, and found a median metallicity 

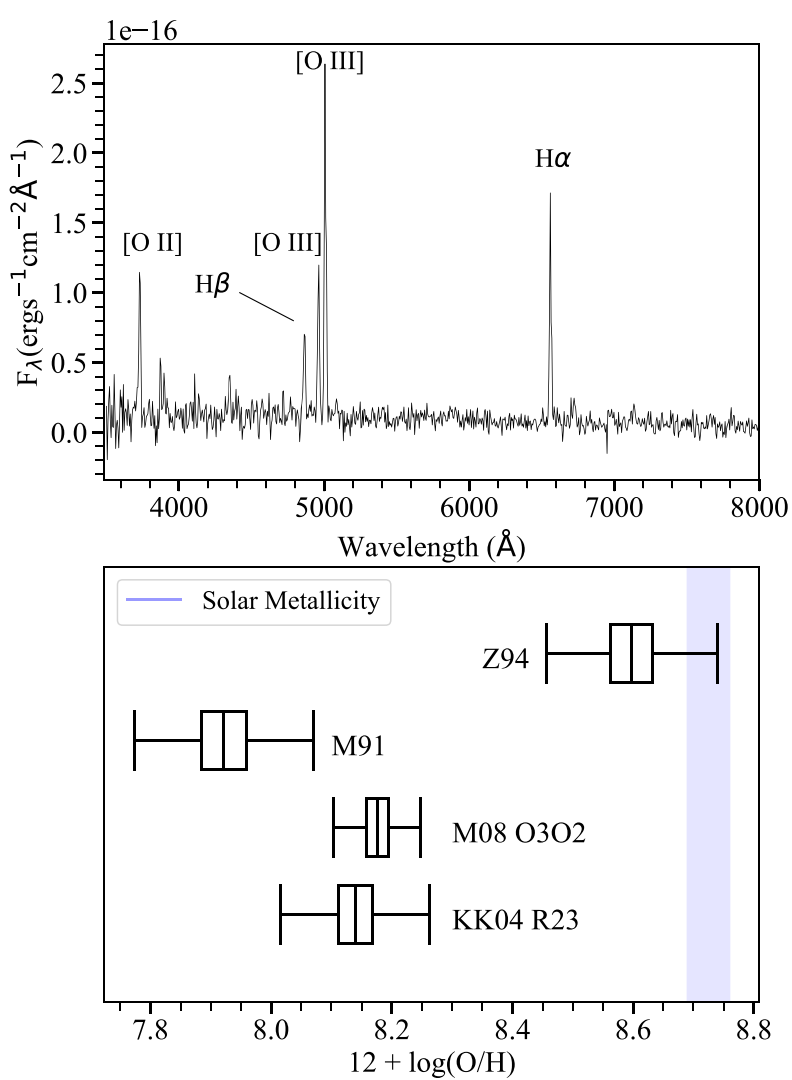

Figure 2. Top panel: NTT galaxy spectrum used as input for pyMCZ, with the relevant lines labelled. The $\mathrm{H} \alpha / \mathrm{H} \beta$ ratio is $2.2 \pm 0.1$. The wavelength is in the rest frame. Bottom panel: Reproduced output of pyMCZ, the metallicity measured via several markers is displayed as box plots. The central value is the median (or 50th percentile). The inner box represents the inter-quartile range (IQR) - 50th to 16th percentile and 84th to 50th percentile (the 16 per cent is an analogy to the Gaussian $1 \sigma$ interval), whilst the outer bars represent the minimum and maximum data values, excluding outliers. The outliers are those values further than 1.5xIQR from the edges of the IQR. The blue band is a range of solar metallicity values found in literature - from 8.69 in Asplund et al. (2009) to 8.76 in Caffau et al. (2011).

Table 1. Galaxy properties from PROSPECTOR for SN $2019 \mathrm{hcc}$, and median values from Schulze et al. (2020) for SLSNe I and SNe II, excluding $12+\log (\mathrm{O} / \mathrm{H})$ for Type II which is from Galbany et al. (2018).

\begin{tabular}{lrrr}
\hline Property & \multicolumn{1}{c}{ SN 2019hcc } & \multicolumn{1}{c}{ SLSN I } & \multicolumn{1}{c}{ SN II } \\
\hline $\log \left(M / \mathrm{M}_{\odot}\right)$ & $7.95_{-0.33}^{+0.10}$ & $8.15_{-0.24}^{+0.23}$ & $9.65 \pm 0.05$ \\
SFR $\left(\mathrm{M}_{\odot} \mathrm{yr}^{-1}\right)$ & $0.07_{-0.01}^{+0.04}$ & $0.59_{-0.20}^{+0.22}$ & $0.58 \pm 0.05$ \\
$\log (\mathrm{sSFR})\left(\mathrm{yr}^{-1}\right)$ & $-9.10_{-1.78}^{+1.42}$ & $-8.34_{-0.32}^{+0.30}$ & $-9.86 \pm 0.02$ \\
Age $(\mathrm{Myr})$ & $2971_{-2131}^{+2019}$ & $427_{-124}^{+119}$ & $4074 \pm 188$ \\
$E(B-V)$ & $0.04_{-0.03}^{+0.06}$ & $0.31_{-0.04}^{+0.05}$ & $0.14 \pm 0.01$ \\
$12+\log (\mathrm{O} / \mathrm{H})$ & $8.08 \pm 0.05$ & $8.26_{-0.30}^{+0.26}$ & $8.54 \pm 0.04$ \\
$M_{B}(\mathrm{mag})$ & $-15.80 \pm 0.20$ & $-17.51_{-0.28}^{+0.30}$ & $-19.15 \pm 0.09$ \\
\hline
\end{tabular}

$12+\log (\mathrm{O} / \mathrm{H})=8.26_{-0.30}^{+0.26}$ for a sample of 37 SLSNe I. Galbany et al. (2018) presented a compilation of 232 SN host galaxies, of which 95 were Type II hosts with an average metallicity $(12+\log (\mathrm{O} / \mathrm{H}))$ of $8.54 \pm 0.04$. The mean metallicity for SN 2019 hcc is within the range of the SLSN I host metallicity found by Schulze et al. (2020), and is low compared to the average metallicity of Type II hosts.

The host galaxy absolute magnitude was measured to be $-15.8 \pm 0.3$ in $r$-band and $-15.8 \pm 0.2$ in $B$-band. Gutiérrez et al.

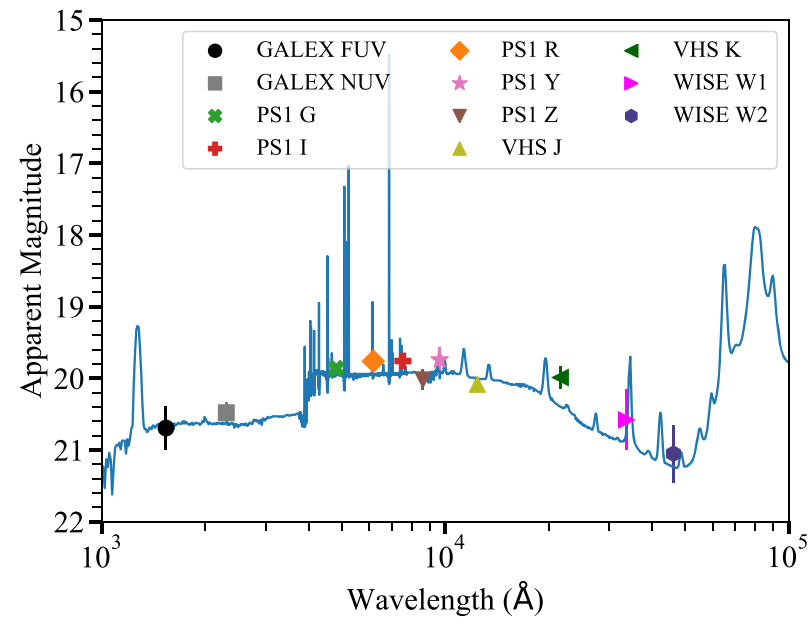

Figure 3. Galaxy photometry of SN 2019hcc from GALEX, PS1, VHS, and WISE, with the best-fitting SED from Prospector, The median $\chi^{2}$ divided by the number of filters (n.o.f.) is 10.65/11 and includes emission lines from H II regions in the fitting.

(2018) defined a faint host as having $M_{r} \gtrsim-18.5 \mathrm{mag}$, and analysed the hosts of a sample of low-luminosity SNe II, finding a mean host luminosity of $-16.42 \pm 0.39 \mathrm{mag}$. Anderson et al. (2016) examined a sample of $\mathrm{SNe}$ II in a variety of host types and found a mean host luminosity $M_{r}$ of $-20.26 \pm 0.14$ mag. For SLSNe I, Lunnan et al. (2014) found a low average magnitude $\left(M_{B} \approx-17.3 \mathrm{mag}\right)$. Table 1 also contains the average $M_{B}$ magnitudes for both SLSNe I and SNe II from Schulze et al. (2020). SN 2019hcc has a lower luminosity and metallicity host with respect to the average value for SNe II and SLSNe I reported in the literature (see Table 1).

We retrieved further SN 2019hcc host galaxy properties by modelling the spectral energy distribution (SED) using the software package Prospector version 0.3 (Leja et al. 2017; Johnson et al. 2019). An underlying physical model is generated using the Flexible Stellar Population Synthesis (FSPS) code (Conroy, Gunn \& White 2009). A Chabrier initial mass function (Chabrier 2003) is assumed and the star formation history ( $\mathrm{SFH}$ ) is approximated by a linearly increasing SFH at early times followed by an exponential decline at late times (functional form $t \times \exp (-t / \tau)$ ). The model was attenuated with the Calzetti et al. (2000) mode, and a dynamic nested sampling package density (Speagle 2020) was used to sample the posterior probability function. To interface with FSPS in PYTHON, PYTHON-fsps (ForemanMackey, Sick \& Johnson 2014) was used.

The photometry images were sourced from the Panoramic Survey Telescope and Rapid Response System (Pan-STARRS, PS1) Data Release 1 (Chambers et al. 2016), the Galaxy Evolution Explorer (GALEX) general release 6/7 (Martin et al. 2005), the ESO VISTA Hemisphere Survey (McMahon et al. 2013), and pre-processed WISE images (Wright et al. 2010) from the unWISE archive (Lang 2014). ${ }^{3}$ The unWISE images are based on the public WISE data and include images from the ongoing NEOWISE-Reactivation mission R3 (Mainzer et al. 2014; Meisner, Lang \& Schlegel 2017). The host brightness was measured using LAMBDAR $^{4}$ (Lambda Adaptive Multi-Band Deblending Algorithm in R; Wright et al. 2016) and the methods described in Schulze et al. (2020).

Fig. 3 shows the best fit SED to the SN 2019hcc photometry for fil-

\footnotetext{
${ }^{3}$ http://unwise.me

${ }^{4}$ https://github.com/AngusWright/LAMBDAR
} 

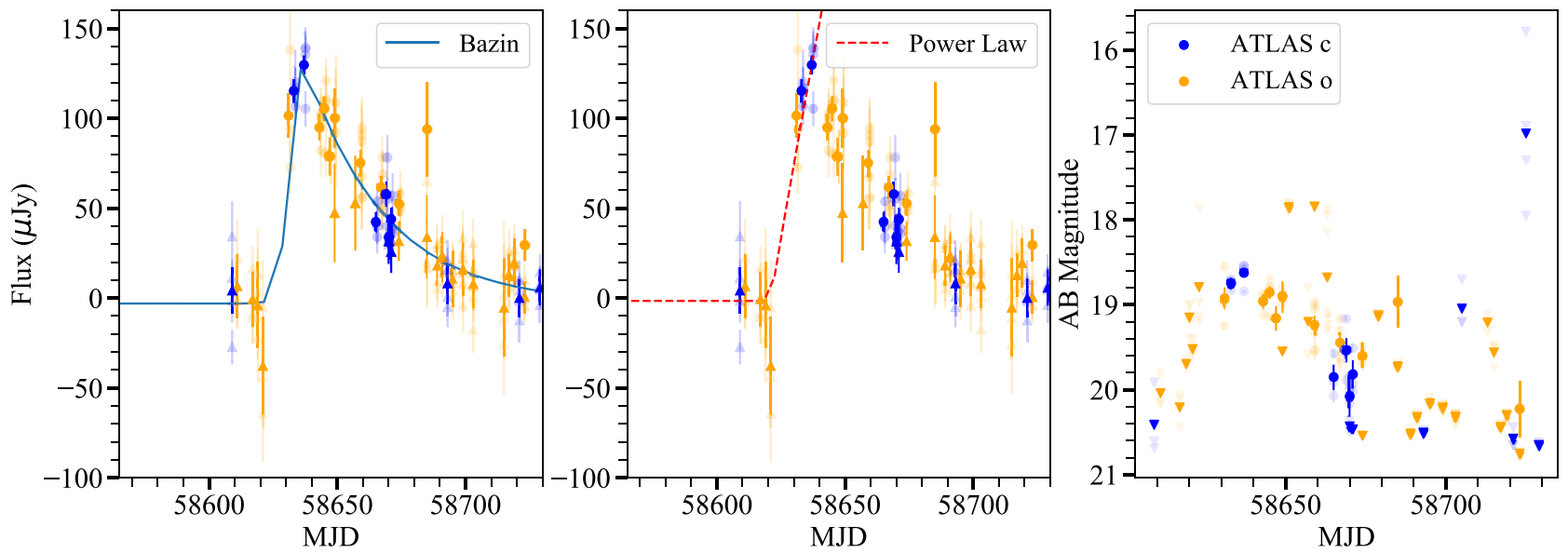

Figure 4. Left-hand panel: fit to the ATLAS forced photometry weighted mean flux (Bazin et al. 2009), in order to determine the peak epoch. This finds the maximum epoch to be MJD 58636.2 \pm 2.2 . Points with errors $>30 \mu \mathrm{Jy}$ have been removed for clarity. Middle panel: a power law fit to the pre-peak flux data (including the upper limits) - this finds an explosion epoch of MJD 58621.0 \pm 7.2. Upper limits are marked as triangles. Where multiple points from the same epoch were taken, these were averaged - the original points are marked with a lighter hue. Right-hand panel: ATLAS forced photometry weighted mean flux converted to $A B$ magnitude, for orange and cyan filters. Images with flux significance $<3 \sigma$ were converted to upper limits.

ters GALEX FUV (20.69 $\pm 0.30 \mathrm{mag})$ and $N U V(20.48 \pm 0.14 \mathrm{mag})$, PS1 GIRYZ $(19.86 \pm 0.03,19.76 \pm 0.04,19.76 \pm 0.04,19.74 \pm 0.17$, $20.02 \pm 0.14 \mathrm{mag})$, VHS $J K(20.08 \pm 0.08,19.99 \pm 0.16 \mathrm{mag})$ and WISE W1 (20.58 $\pm 0.42 \mathrm{mag})$ and $W 2(21.05 \pm 0.40 \mathrm{mag})$. The magnitudes are in the $\mathrm{AB}$ system and corrected for Milky Way extinction. Table 1 shows the galaxies properties inferred from the best-fitting SED to the host galaxy photometry. The $E(B$ $-V$ ) inferred for SN 2019hcc matches well with the $E(B-V)$ based on the Milky Way extinction. The mass of the host best matches the median SLSN I host mass, whilst the SFR is low for both SLNSe I and SNe II. The age of the SN 2019hcc host has a large uncertainty that covers the range of both classes, and the magnitude is low for both classes. The SFR is significantly lower for SN 2019hcc. However, the mass of the host is lower than the median for both SLSNe I and SNe II, and therefore the sSFR falls between the two.

\section{PHOTOMETRY}

\subsection{Rise time and explosion epoch}

We determined the rise time and explosion epoch following the methodology presented in González-Gaitán et al. (2015). We applied this approach to the ATLAS data only, both orange and cyan, as it is the only photometry available which covers the pre-peak light curve albeit with many upper limits. It is not ideal to combine different bands, however, as there are few points it is an unavoidable uncertainty. We then measure the explosion epoch using a power-law fit (equation 2) from the earliest pre-peak upper limit to maximum luminosity:

$$
\begin{array}{r}
f(t)=a\left(t-t_{\exp }\right)^{n} \text { if } t>t_{\exp } \\
f(t)=0 \text { if } t<t_{\exp } .
\end{array}
$$

Here, $a$ is a constant and $n$ is the power index, both of which are free parameters, and $t_{\exp }$ is the explosion date in days. This fit was done using a least-squares fit as implemented by SCIPY.CURVE_FIT in PYTHON to the pre-maximum light curve in flux, and the explosion epoch was measured to be MJD 58621.0 \pm 7.2. An alternative method of measuring the explosion epoch is to take the midpoint between the first non-detection and the first detection - this would be between MJD 58609 and MJD 58631, giving an estimate of the explosion epoch of MJD 58620, which is within the errors and consistent with the previous measurement.

For the epoch of maximum light, we used the phenomenological equation for light curves from Bazin et al. (2009). This form, as shown in equation (3), has no physical motivation but rather is flexible enough to fit the shape of the majority of supernova light curves.

$f(t)=A \frac{e^{-\left(t-t_{0}\right) / t_{\text {fall }}}}{1+e^{\left(\mathrm{t}-\mathrm{t}_{0}\right) / \mathrm{t}_{\text {rise }}}}+B$

Here, $t_{0}, t_{\text {rise }}, t_{\text {fall }}, A$, and $B$ are free parameters. The derivative, as seen in equation (4), was used to get the maximum epoch $\left(t_{\max }\right)$, and the uncertainties from the fit were propagated through the below equation (González-Gaitán et al. 2015):

$t_{\text {max }}=t_{0}+t_{\text {rise }} \times \log \left(\frac{-t_{\text {rise }}}{t_{\text {rise }}+t_{\text {fall }}}\right)$.

The maximum epoch was found from the Bazin fit to be MJD 58636.2 \pm 2.2 - this was done by fitting to the flux data, see the right-hand panel on Fig. 4. This will be the maximum hereafter referred to in the paper, and can be approximated as the peak in ATLAS $o$-band, as this is the band the majority of these points are in. Points with an error greater than $30 \mu \mathrm{Jy}$ have been removed for clarity. Combining this result with the explosion epoch gives a rise time of $15.2 \pm 7.5 \mathrm{~d}$.

ATLAS $o$-band is close to $R$-band. The average $R$-band rise from the 'gold' samples (consisting of 48 and $38 \mathrm{SNe}$ each from different surveys) of SNe II from González-Gaitán et al. (2015) was $14.0_{-9.8}^{+19.4}$ d. Pessi et al. (2019) reported an average $r$-band rise time for a sample of $73 \mathrm{SNe}$ II of $16.0 \pm 3.6 \mathrm{~d}$. Both results are consistent with our measured value - therefore it seems the rise of SN 2019hcc is typical for a SN II. In contrast, SLSNe I light curves have longer time-scales with an average rise of 28 and $52 \mathrm{~d}$ for SLSNe I Fast and Slow, respectively (Nicholl et al. 2015; Inserra 2019). Despite the average longer rise of SLSNe I to SNe II, it should be noted that the fastest riser SLSNe I can have some overlap within the errors of the slowest SNe II values from González-Gaitán et al. (2015). 


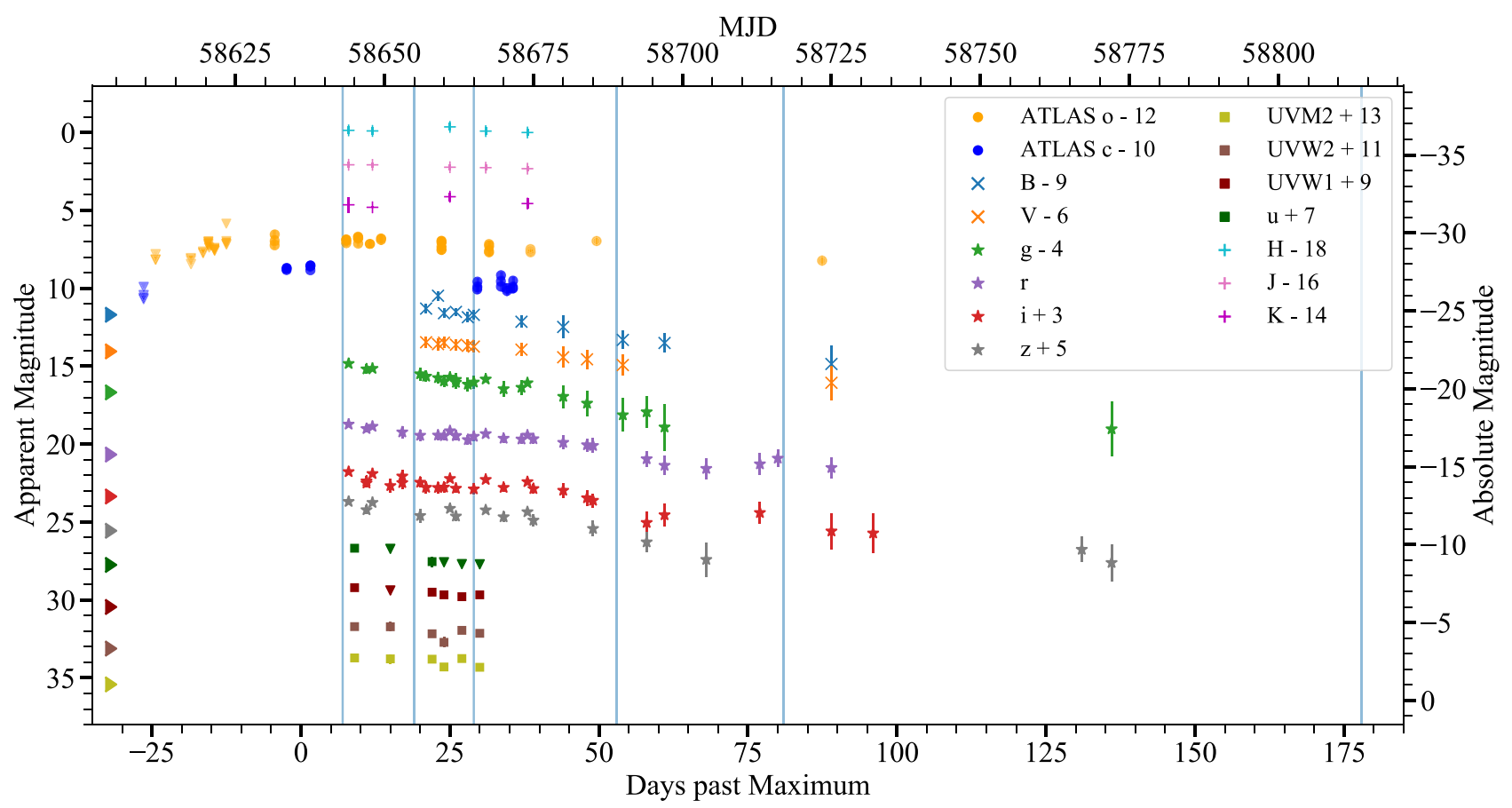

Figure 5. Photometry for SN 2019hcc - the light curves from various sources: BVgri, bands were taken by LCO, and griz bands were also taken by LT. Alongside this, there is ATLAS data including the pre-peak limits, Swift UV data, and GROND NIR data. The vertical lines mark the epochs when the spectra were taken. The markers on the left $y$-axis signify the galaxy magnitude in the respective bands.

\subsection{Multiband light curve}

The majority of photometric data were taken by LCO in bands $B V g r i$, and by LT in bands griz. The light curve produced from this data was created using a code written using PYTHON packages AstroPy and PhotUtils (see Appendix A for further detail). This was complemented by ATLAS data in the orange and cyan bands, UV data from Swift, optical, (griz) and NIR (JHK) data from GROND. Fig. 5 shows the photometric evolution of SN 2019hec in all available bands. The UV data covers $21 \mathrm{~d}$, and appears to follow a linear decline. The NIR data covers a similar period of $30 \mathrm{~d}$, and are roughly constant in magnitude. There is a linear decline of $\sim 50 \mathrm{~d}$ from peak in all optical bands, with a magnitude change of $\sim 1.5 \mathrm{mag}$ in $r$-band, followed by a steeper drop of $\sim 2 \mathrm{mag}$ from 50 to $70 \mathrm{~d}$. The decline rate is similar in the other bands with the exception of $g$-band which declines faster, at a rate of $\sim 2 \mathrm{mag}$ in the first $\sim 50 \mathrm{~d}$ after maximum light, and subsequently $\sim 3$ mag in the steeper decline. The $B V$-bands data for Swift were excluded as they were contaminated by host galaxy light. Such a contamination is far less in $u, u v w 1, u v m 2$, and $u v w 2$. The Swift detections were at level of 3$4 \sigma$. GROND griz magnitudes were not template subtracted as there were no templates available. However, the data were taken soon after maximum light, where the difference between the host galaxy magnitude and that of SN 2019hcc is at its maximum, and therefore should not add significant uncertainty. LT and LCO magnitudes were template subtracted as part of the photometry code described in Appendix A.

Fig. 6 shows the evolution of the blackbody temperature fit to the photometric data together with the $B-V$ colour evolution, both for SN 2019hcc and a selection of SNe II. These are: SN 2013ej (Valenti et al. 2014), SN 2014G (Terreran et al. 2016), SN 2008fq (Taddia et al. 2013), SN 1998S (Fassia et al. 2000, 2001), SN 2009dd, and SN 2010aj (Inserra et al. 2013a) together with a sample of 34 SNe II from Faran et al. (2014). SNe 1998S and 2014G - a Type IIn and
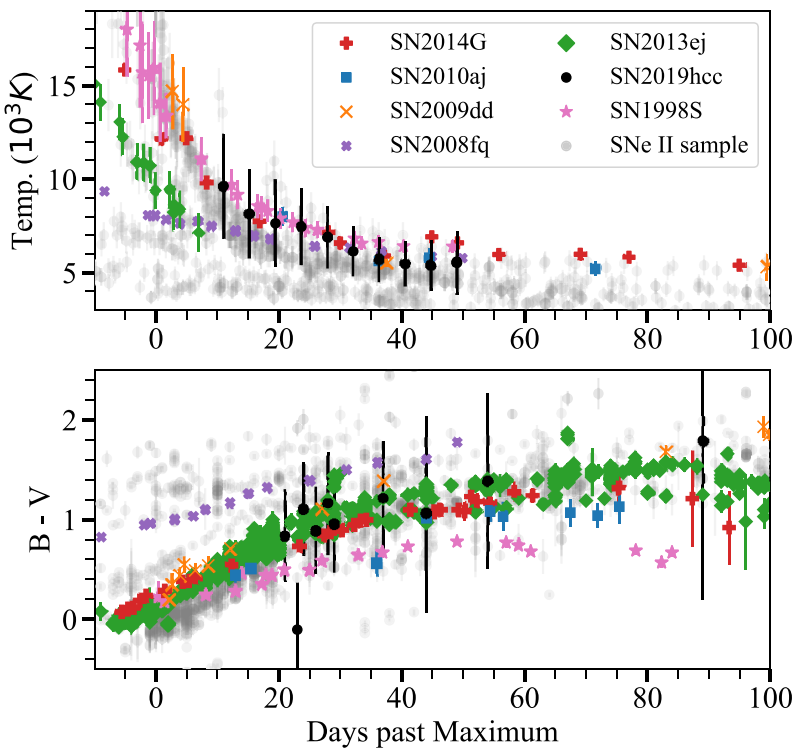

Figure 6. Top panel: the blackbody temperature evolution - for SN $2019 \mathrm{hcc}$ this is from the fit to the photometric data, whilst for the other $\mathrm{SNe}$ it is from the literature. The uncertainties for SN 2019hcc are from the curve fit. There were no uncertainties reported in the literature for the temperatures of SN 2014G and SN 2008fq. Bottom panel: colour evolution $B-V$ compared with the same $\mathrm{SNe}$ of the upper panel. The temperature and colour evolution from the sample of SNe II from Faran et al. (2014) is shown in grey.

IIL, respectively - were chosen for their spectroscopic similarity to SN 2019hcc near peak. SN 2013ej, SN 2010aj, and SN 2008fq provide a small sample of well-observed SNe II displaying a similar peak magnitude of SN 2019hcc, which fall in the category of relatively bright Type II (Inserra et al. 2013a). The griz bands for 
SN 2019hcc were individually interpolated to 10 evenly spaced points across the date range, and the temperature was found by fitting to these bands at each point. The interpolation was done using Gaussian processes from SKLEARN, and the errors from the photometric points were interpolated using interp1d from SCIPY. For the colour, the points were chosen where both $B$ and $V$ were available. The fits for temperature are expected to become worse as the photospheric phase passes and the blackbody approximation is less appropriate. The temperature and colour evolution for the Type II supernovae were taken from the above papers. The temperature and colour evolution of the SNe II sample (Faran et al. 2014) were calculated from the data available on the Open Supernova Catalogue (Guillochon et al. 2017). These SNe have a large range within which the temperature evolution falls, and appears to have multiple branches, which spans the range of temperature and colour evolution of the SNe II selected for a direct comparison. From Fig. 6, it appears the colour and temperature evolution of SN 2019hcc is not unusual with respect to the $\mathrm{SNe}$ chosen for a direct comparison or that of Faran et al. (2014). Overall, SN 2019hcc colour and temperature evolution appears to closely resemble those of SN 2014G and SN 2009dd. The colour evolution appears to have two regimes, a steeper slope until $\sim 30-40 \mathrm{~d}$ followed by a less steep rise. The first slope is $2.8 \mathrm{mag}$ per $100 \mathrm{~d}$ which is very similar to the average 2.81 mag per $100 \mathrm{~d}$ obtained by de Jaeger et al. (2018) for $B-V$. They also found a transition between the two regimes at $37.7 \mathrm{~d}$ which is roughly consistent with what is seen in the colour evolution.

As the 'w' shape profile of SN 2019hcc first spectrum is similar to that observed in SLSNe I, in Fig. 7, we also compare the temperature evolution of SN 2019hcc with a sample of SLSNe I: iPTF16bad (Yan et al. 2017), SN 2010kd (Kumar et al. 2020), PTF12dam (Nicholl et al. 2013), and LSQ14mo (Leloudas et al. 2015b; Chen et al. 2017b). We selected this small subset of SLSNe I mainly due to the spectral similarity, see Section 7 for further information. We also compare to an average temperature evolution for SLSNe I (Inserra et al. 2017, and reference therein), similarly to what was previously done with SNe II. LSQ14mo is the only SLSNe I with a similar temperature evolution to SN 2019 hcc.

\section{LIGHT-CURVE ANALYSIS}

\subsection{Bolometric light curve}

We created a pseudo-bolometric light curve from an SED fit to the available photometry, which was interpolated according to the chosen reference band. We used the SDSS $r$-band and the ATLAS $o$-band as reference, as these bands should approximately cover a similar region of the electromagnetic spectrum, to cover as many epochs as possible. Each band was integrated using the trapezium rule. The redshift, distance, and reddening used were reported in Section 2.

The light-curve evolution of SNe II was considered quantitatively by Anderson et al. (2014b) and Valenti et al. (2016). The decline of the initial steeper slope of a light curve and the second shallower slope can be described as S1 and S2, respectively - in SNe IIL these are very similar or the same (Anderson et al. 2014b). S1 and S2 were originally described for $V$-band; however, Valenti et al. (2016) also performs this analysis for pseudo-bolometric light curves and the key parameters are very similar - and in fact the transition between the early fast slope S1 and the shallow late slope S2 is more evident in pseudo-bolometric curves (Valenti et al. 2016). S2 is followed by the plateau-tail phase (Utrobin 2007), also known as the postrecombination plateau (Branch \& Wheeler 2017), which drops into the ${ }^{56} \mathrm{Co}$ tail. The formalism reported in Valenti et al. (2016) can be

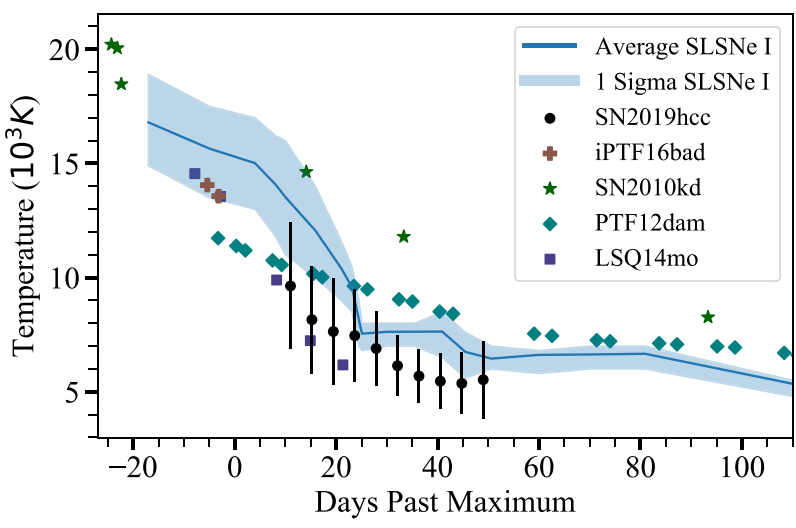

Figure 7. Temperature comparison of SN 2019hcc with a small sample of SLSNe I. The closest SLSN I in temperature to SN 2019hcc at the epoch of $+7 \mathrm{~d}$ is LSQ14mo. The SLSN temperatures are taken from the literature (see text). The average temperature for SLSNe I is taken from Inserra et al. (2017) and reference therein.

described by the following equation:

$f(t)=\frac{-A_{0}}{1+e^{\left(t-t_{\mathrm{pt}}\right) / w_{0}}}+\left(t \times p_{0}\right)+m_{0}$.

Here, the variables $A_{0}, w_{0}, m_{0}$ are free parameters describing the shape of the drop, $p_{0}$ describes the decline of the tail, and $t_{\mathrm{pt}}$ describes the length of the plateau, measured from the explosion to the midpoint between the end of the plateau phase and start of the radioactive tail.

The top panel on Fig. 8 shows the pseudo-bolometric light curve, however, there is no distinguishable change in the slope leading to a clear distinction of S1 and S2, and after approximately $60 \mathrm{~d}$ past maximum the light curve transits into a 'plateau-tail phase' and then drops into a radioactive tail. As there are not multiple slopes in the initial decline, S1 and S2 will hereafter be collectively referred to as S2 for SN 2019hcc, leading to a Type IIL sub-classification for the supernova. The $\mathrm{S} 2$ decline was found to be $1.51 \pm 0.09 \mathrm{mag}$ per $50 \mathrm{~d}$. The best-fit $t_{\mathrm{pt}}$ was $66.0 \pm 1.1 \mathrm{~d}$, and $p_{0}$ was measured via a linear fit and found to be $1.38 \pm 0.49 \mathrm{mag}$ per $100 \mathrm{~d}$. Valenti et al. (2016) found a mean length of the plateau in SNe II of $t_{\mathrm{pt}}=100$, which is up to the mid-way in the plateau-tail phase. Considering this average, SN 2019hcc has a relatively short plateau duration, which could suggest a lower ejecta mass, but could also be due to a smaller progenitor radius or a higher explosion energy (Popov 1993). This fitting was performed for the pseudo-bolometric light curve rather than $V$-band due to the sparsity of photometric data in this band, particularly for the tail of the light curve.

The middle panel of Fig. 8 shows the full bolometric light curve - this was found by fitting a blackbody to the photometry and integrating between 200 and $25000 \AA$. The bolometric light curve required interpolation and extrapolation of additional points for epochs where some bands were not observed. This was done by taking a constant colour from the nearest points in the other bands however this is an assumption which increases the uncertainty in the resultant curve. The tail luminosity $L_{\text {tail }}$ is marked, and a ${ }^{56} \mathrm{Co}$ tail has been plotted using equation (6), as from Jerkstrand et al. (2012), which gives the bolometric luminosity for the theoretical case of a fully trapped ${ }^{56}$ Co decay.

If full trapping of gamma-ray photons from the decay of ${ }^{56} \mathrm{Co}$ is assumed, the expected decline rate is $0.98 \mathrm{mag}$ per $100 \mathrm{~d}$ in $\mathrm{V}$ band (Woosley, Pinto \& Hartmann 1989; Anderson et al. 2014b). The tail of SN 2019 hcc clearly declines faster than the ${ }^{56}$ Co tail as 


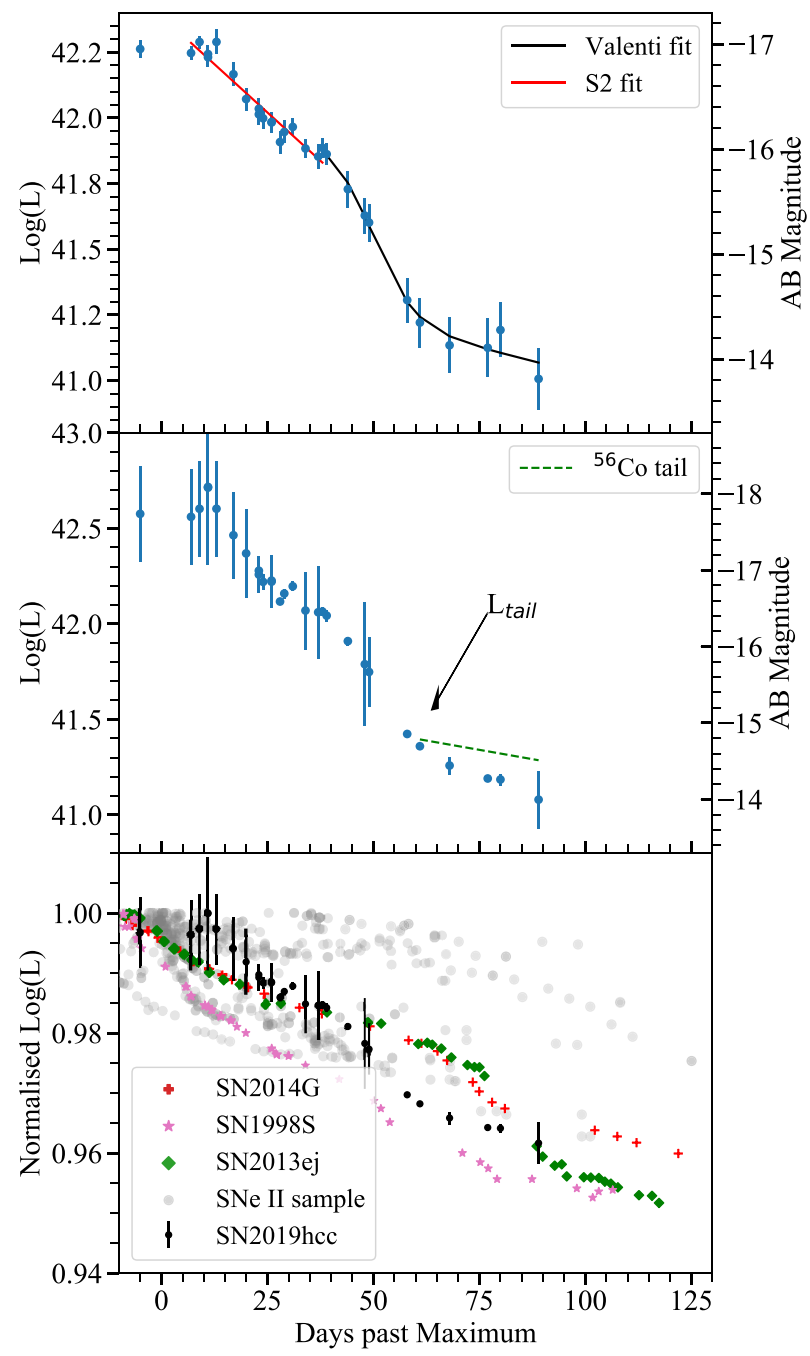

Figure 8. Top panel: the pseudo-bolometric light curve of SN 2019hcc, with $r$-band as the reference. Middle panel: the bolometric light curve of SN 2019hcc. The tail magnitude and comparison to ${ }^{56} \mathrm{Co}$ decay rate is marked. Bottom panel: the bolometric light curve of SN 2019hcc, compared to those of SNe II SN 2014G (Terreran et al. 2016), SN 2013ej (Huang et al. 2015), and SN 1998S (Fassia et al. 2000, 2001). The bolometric light curves of the sample of SNe II from Faran et al. (2014) is shown in grey - two distinct branches can be seen which could be described with the SN IIL and SN IIP subcategories. The light curves have been normalized with respect to the maximum.

shown in the middle panel of Fig. 8. If this is indeed the radioactive tail, it seems that SN 2019hcc displays incomplete trapping. This is not entirely unexpected as Gutiérrez et al. (2017b) showed that most fast-declining $\mathrm{SNe}$ show a tail decline faster than expected from ${ }^{56} \mathrm{Co}$ decay. Terreran et al. (2016) found incomplete trapping for SN 2014G, one of the SNe in our comparison sample. They suggested a few possibilities for incomplete trapping such as a low ejecta mass, high kinetic energy, or peculiar density profiles. However, dust formation could also result in a fast-declining tail, and additional effects such as a different radioactivities could affect the decline (Branch \& Wheeler 2017), as well as CSM-ejecta interaction, which can contribute to the luminosity at late times (e.g. Andrews et al. 2019).

The lower panel of Fig. 8 shows a comparison of the bolometric light curve of SN 2019hcc with Type II SNe 2013ej and 2014G,
Table 2. Here, $V_{50}$ is the $V$-band mag decline in the first $50 \mathrm{~d}$ (roughly equivalent to $\mathrm{S} 2$ ), measured directly from the light curves with a linear fit. Rise times and peak absolute magnitude are in $R$-band for SN 2014G (Terreran et al. 2016) and SN 2013ej (Richmond 2014; Huang et al. 2015), or ATLAS $o$-band for SN 2019hc. Rise time and peak values for SN 1998S are also in the $R$-band, however, they are estimated from the light curve rather than taken from literature. Also shown are the average values for a sample of 10 SN IIL and 18 SN IIP from Faran et al. (2014). Though these populations have been previously discussed as continuous, the distinction is still useful to give context to the measured values. Anderson et al. (2014b) found a mean S2 of 0.64 for a sample of $116 \mathrm{SNe}$ II, roughly the average of the IIL and IIP sub-classes in the above. The rise time for $\mathrm{SNe}$ II is taken from Pessi et al. (2019). The average absolute peak magnitude in $R$-band from SNe II comes from Galbany et al. (2016).

\begin{tabular}{lccc}
\hline SN & \multicolumn{1}{c}{$V_{50}$} & Rise $(\mathrm{d})$ & Peak (Absolute Mag) \\
\hline SN 2019hcc & $1.52 \pm 0.03$ & $15.3 \pm 7.4$ & -17.7 \\
SN 2014G & $1.58 \pm 0.06$ & $14.4 \pm 0.4$ & -18.1 \\
SN 2013ej & $1.24 \pm 0.02$ & $16.9 \pm 1$ & -17.64 \\
SN 1998S & $1.87 \pm 0.07$ & $\sim 18$ & $\sim-18.1$ \\
SNe II & $1.43 \pm 0.21$ (IIL) & $16.0 \pm 3.6$ & $-16.96 \pm 1.03$ \\
& $0.31 \pm 0.11$ (IIP) & & \\
\end{tabular}

and with the Type IIn SN 1998S. These were chosen for comparison as they present a similar photometric evolution to SN 2019hcc (see Section 4). The bolometric light curves from the sample of SNe II from Faran et al. (2014) are also included, and two distinct branches can be seen which would correspond to the historic SN IIL and SN IIP sub-classifications. However, note the small sample size of this study compared with other sample analyses. All light curves have been normalized by the peak luminosity for comparison. This panel supports that the sample of SNe II discussed would all be considered SNe IIL, or fast decliners.

A SN IIL has been defined as where the $V$-band light curve declines by more than 0.5 mag from peak brightness during the first $50 \mathrm{~d}$ after explosion (e.g. Faran et al. 2014). The initial decline of SN 2019hcc was also measured in $V$-band and is displayed, along with other properties, in Table 2 together with the comparison $\mathrm{SNe}$ and the average values for SN IIP and SN IIL. Looking at Fig. 8, the S2 slope of SN 2019hcc appears steeper, and the plateau shorter, than the comparison SNe II SN 2014G and SN 2013ej. However, SN 1998S has a faster intial decline, and appears to transition to the tail at a comparable epoch. SN 2013ej has the most distinct S1 and S2. The radioactive tail of SN 2019hcc shows a similar decline rate to all comparison $\mathrm{SNe}$ which also seem to display incomplete trapping, or at the very least a radioactive tail decay faster than ${ }^{56} \mathrm{Co}$ decay. The SN 2019hcc light-curve evolution drops out of the photospheric phase sooner than SN 2013ej and SN 2014G - implying a lower ejecta mass. It could therefore be suggested that the ejecta mass of SN 2019hcc is lower than the that of these other SNe, however, other factors such as explosion energy could also play a role (Popov 1993).

\section{$5.2{ }^{56} \mathrm{Ni}$ production}

Jerkstrand et al. (2012) presented a method to retrieve the ${ }^{56} \mathrm{Ni}$ mass produced by comparing the estimated bolometric luminosity in the early tail-phase with the theoretical value of fully trapped ${ }^{56} \mathrm{Co}$ deposition, which is given by

$L(t)=9.92 \times 10^{41} \times \frac{M_{56} \mathrm{Ni}}{0.07 \mathrm{M}_{\odot}} \times\left(e^{-t / 111.4 d}-e^{-t / 8.8 d}\right)$,

where $t$ is the time since explosion, $L(t)$ is the luminosity in $\mathrm{erg} \mathrm{s}^{-1}$ at that time, $8.8 \mathrm{~d}$ is the e-folding time of ${ }^{56} \mathrm{Ni}$ and $111.14 \mathrm{~d}$ is the 
e-folding time of ${ }^{56} \mathrm{Co}$ decay. It is also assumed that the deposited energy is instantaneously re-emitted and that no other energy source has any influence. To calculate the mass of ${ }^{56} \mathrm{Ni}$, the tail luminosity and the time at which the tail begins should be used in equation (6).

A visible transition can be seen in Fig. 8 into the tail of SN 2019hcc at $61 \mathrm{~d}$ past maximum therefore we selected the tail luminosity as the magnitude at the point of transition. With this tail magnitude, according to the above approach, the mass of ${ }^{56} \mathrm{Ni}$ is $0.035 \pm 0.008 \mathrm{M}_{\odot}$. The uncertainty was calculated as $0.1 \mathrm{dex}$, as a measure of the distance to the adjacent points, as the exact location of the tail start is uncertain. This is only a lower limit due to likely incomplete trapping. Anderson et al. (2014b) performed this analysis on a large set of $\mathrm{SNe}$ II, and found a range of ${ }^{56} \mathrm{Ni}$ masses from 0.007 to $0.079 \mathrm{M}_{\odot}$, with a mean value of $0.033 \mathrm{M}_{\odot}(\sigma=0.024)$. A survey of literature values led to a mean mass ${ }^{56} \mathrm{Ni}=0.044 \mathrm{M}_{\odot}$ for a sample of 115 SNe II (Anderson 2019). Therefore, we conclude that the value retrieved for SN 2019hcc is within the expected range for a SN II.

\section{SPECTROSCOPY}

Fig. 9 shows the spectral evolution of SN 2019hcc, labelled with the phase with respect to maximum light (MJD 58636). The spectra have been flux-calibrated according to the broad-band photometry. The last epoch was not calibrated according to the photometry as none was available. At $+81 \mathrm{~d}$, the SED no longer follows a blackbody assumption as the ejecta is now optically thin and the photospheric phase is over; however, the blackbody fit to the the photosphere is a valid approximation for the earlier spectra. The light-curve analysis from Section 5 suggests the end of the plateau/photospheric phase, $t_{\mathrm{pt}}$, at approximately $+66 \mathrm{~d}$ from explosion. Emission lines from the host galaxy can be seen, particularly from $+53 \mathrm{~d}$. The resolution of the spectra can be found in Appendix B.

The spectra were also corrected for redshift and de-reddened according to the Cardelli Extinction law using Av $=0.19$ mag and $\mathrm{Rv}=3.1$ (Cardelli, Clayton \& Mathis 1989). They have been offset for clarity on an arbitrary $y$-axis. The flux has been converted to $\log \left(F_{v}\right)$ where $F(v)=F(\lambda) \lambda^{2} / 3 e 18$ to highlight the absorption features.

As can be seen, the first spectrum at $+7 \mathrm{~d}$ after peak displays a ' $w$ '-shaped profile at the rest-wavelengths typical of $\mathrm{O}$ II lines with absorption minima at approximately 4420 and $4220 \AA$, which originally motivated the classification as a SLSN I. However, these signatures disappear in subsequent spectra with the $\mathrm{H} \alpha$ emission becoming the dominant spectral feature. Aside from the w-shape, the first spectrum is relatively featureless. A well developed $\mathrm{H} \alpha$ profile can be seen from $+19 \mathrm{~d}$, as well as $\mathrm{H} \beta$ and $\mathrm{H} \gamma$, though less developed Balmer lines can also be seen at $+7 \mathrm{~d}$. Fe II and $\mathrm{He}$ I lines can also be seen from the $+7 \mathrm{~d}$ spectrum and become welldeveloped by $+19 \mathrm{~d}$. The typical core-collapse $\mathrm{SN}$ forbidden lines of [O I] at $\lambda \lambda 6300,6363$ and [Ca II] at $\lambda \lambda 7291,7323$ are not seen despite SN 2019hcc appearing to reach the nebular phase, which roughly starts at 100-200 d (Fransson \& Chevalier 1989). There could be a few possibilities for their absence. The first is that the nebular phase has not been reached. Alternatively, as the strength of [O I] increases with the ZAMS mass (e.g. Dessart \& Hillier 2020), it would imply a ZAMS mass of the SN 2019hcc progenitor sufficiently low that the [OI] are not visible. Another possibility is that SN 2019hcc is too faint with respect to the host and the lines have not yet developed.

In SN 2014G, after $\sim 80$ d the emission feature of [Ca II] at $\lambda \lambda 7291$, 7323 starts to become visible, approximately coincident with the sudden drop in the light curve (Terreran et al. 2016). SN 2013ej also

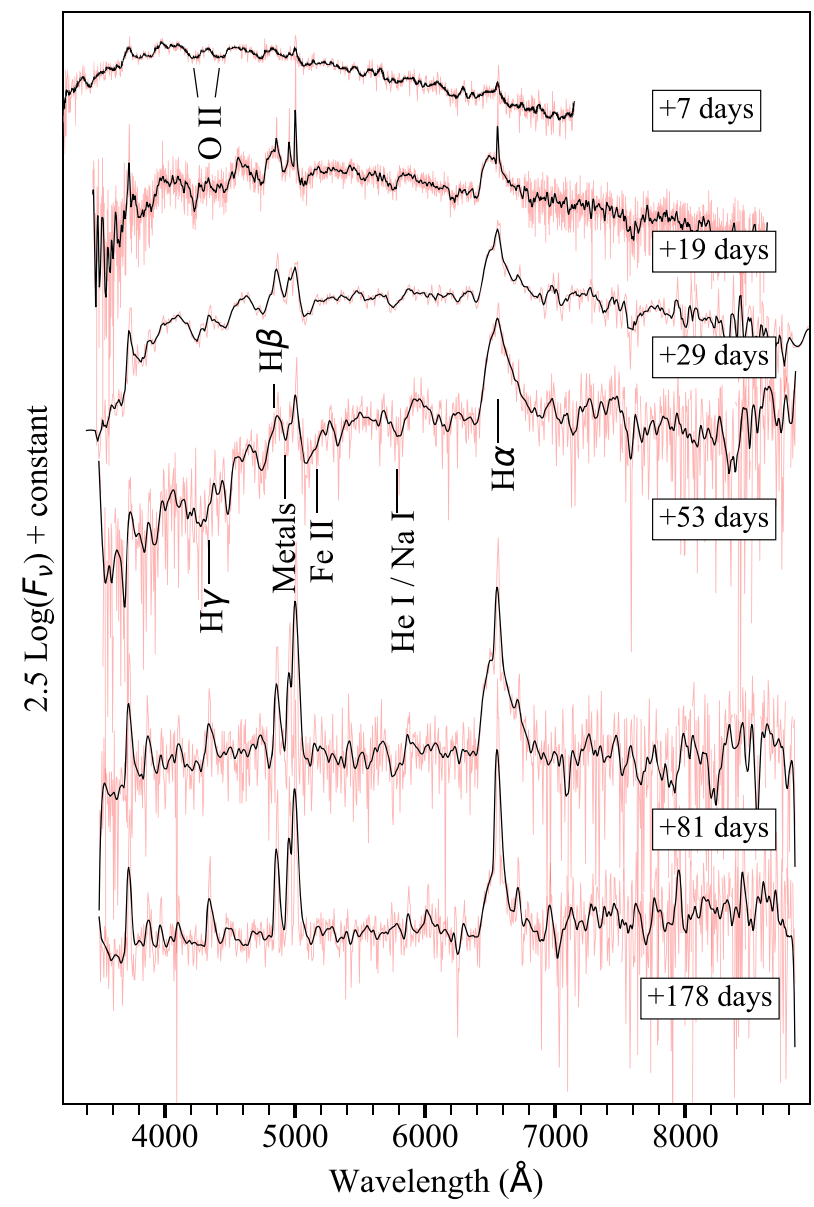

Figure 9. The spectra for SN 2019hcc and their phase with respect to maximum light (MJD 58636). The wavelength is in the rest frame. The spectra have been corrected according to the photometry (excluding the last epoch which had no photometry available), de-reddened, and redshift corrected. They have also been smoothed using a moving average - this recalculates each point as the average of those on either side, in this case for five iterations - (black) with the original overlaid (red). The flux has been converted to $\log \left(F_{v}\right)$ to emphasize absorption features. The most prominent elements have been labelled - here 'Metals' refers to a combination of Ba II, Sc II, and Fe II.

shows [Ca II] and [OI] forbidden lines from $109 \mathrm{~d}$, when the SN entered the nebular phase (Bose et al. 2015), suggesting SN 2019hcc is unusual in this respect. However, Branch \& Wheeler (2017) noted the spectra of some SN IIL (e.g. SN 1986E, SN 1990K) do not contain the standard emission lines of core-collapse supernovae, and the forbidden lines arising in the ejecta may be suppressed by high densities or obscured by the circumstellar medium (CSM) that produces the extended hydrogen emission.

The flux of $\mathrm{H} \alpha$ in the $+178 \mathrm{~d}$ spectrum (excluding the narrow host contribution component) is $\sim 5$ times that of $\mathrm{H} \beta$. For case $\mathrm{B}$ recombination in the temperature regime $2500 \leq T(\mathrm{~K}) \leq 10000$ and electron density $10^{2} \leq n_{\mathrm{e}} \leq 10^{6}$, the $\mathrm{H} \alpha$ line should be 3 times stronger than $\mathrm{H} \beta$ (Osterbrock \& Ferland 2006). However, the case $\mathrm{B}$ recombination is not observed in SNe II before a couple of years. Kozma \& Fransson (1998) suggested at 200 d past explosion in SN 1987A this ratio should have been around 5, based on the total calculated line flux and using a full hydrogen atom with all nl-states up to $n=20$ included. The ratio of SN 2019hec appears similar to SN 1987A and other SNe II at the onset of the nebular phase. Despite the $\mathrm{H} \alpha / \mathrm{H} \beta$ ratio being higher than the case $\mathrm{B}$ recombination, it is 


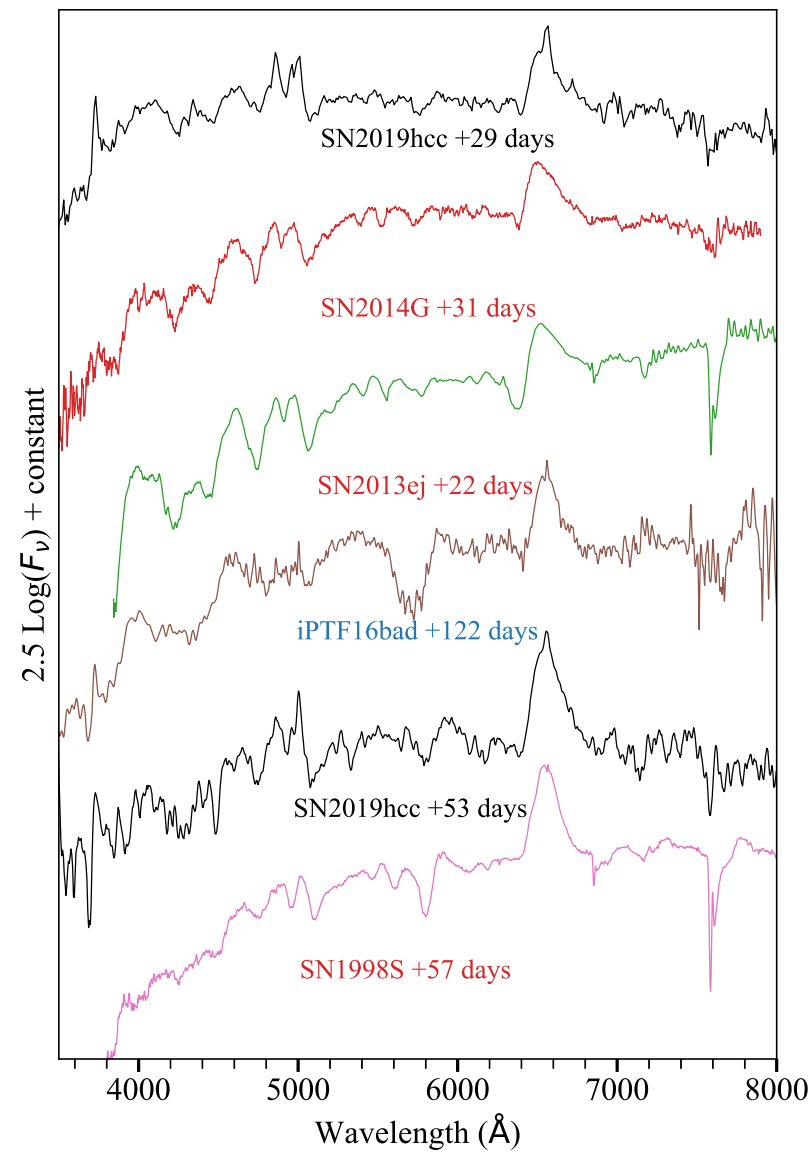

Figure 10. SN $2019 \mathrm{hcc}$ at +29 d post peak is compared to moderately luminous Type II SN 2014G and SN 2013ej, and SLSN I iPTF16bad which displays $\mathrm{H} \alpha$ at late time (at $+100 \mathrm{~d}$ post-peak) in its spectra. SN 2019hec at $+53 \mathrm{~d}$ past peak is also compared to SN 1998S. The wavelength is in the rest frame. Text in red refers to Type II, while in blue to the only SLSN I.

still sufficiently low that we can conclude that any additional flux to $\mathrm{H} \alpha$ should be insignificant. Excess flux in $\mathrm{H} \alpha$ could be a clue that $\mathrm{H} \alpha$ is also collisionally excited, suggesting interaction (Branch et al. 1981). As the $\mathrm{H} \alpha$ profile evolves it appears to become asymmetrical, suggesting a multicomponent fit in the late spectra. The simplest explanation for this is that a mostly spherical ejecta is interacting with a highly asymmetric, hydrogen-rich CSM (Benetti et al. 2016). This is in contrast with the quick decay of the tail, suggesting that such asymmetry might be intrinsic of the ejecta or the result of other lines that are not resolved, for example $[\mathrm{N} \mathrm{II}] \lambda 6584$. An asymmetric line profile can also be interpreted as evidence for dust formation in the ejecta (e.g. Smith, Foley \& Filippenko 2008).

\subsection{Spectral comparison}

Comparison of SN 2019hcc with the moderately luminous SNe II (Inserra et al. 2013a) reported in Section 5 and Fig. 6, together with SLSN I iPTF16bad, is shown in Fig. $10 .^{5}$

iPTF16bad at late times displays $\mathrm{H} \alpha$ emission due to the collision with a $\mathrm{H}$-shell ejected approximately $30 \mathrm{yr}$ prior, thought to be due to pair instability pulsations (Yan et al. 2015, 2017), and merits

${ }^{5}$ These spectra were taken from the Weizmann Interactive Supernova Data Repository (WISeREP; Yaron \& Gal-Yam 2012)

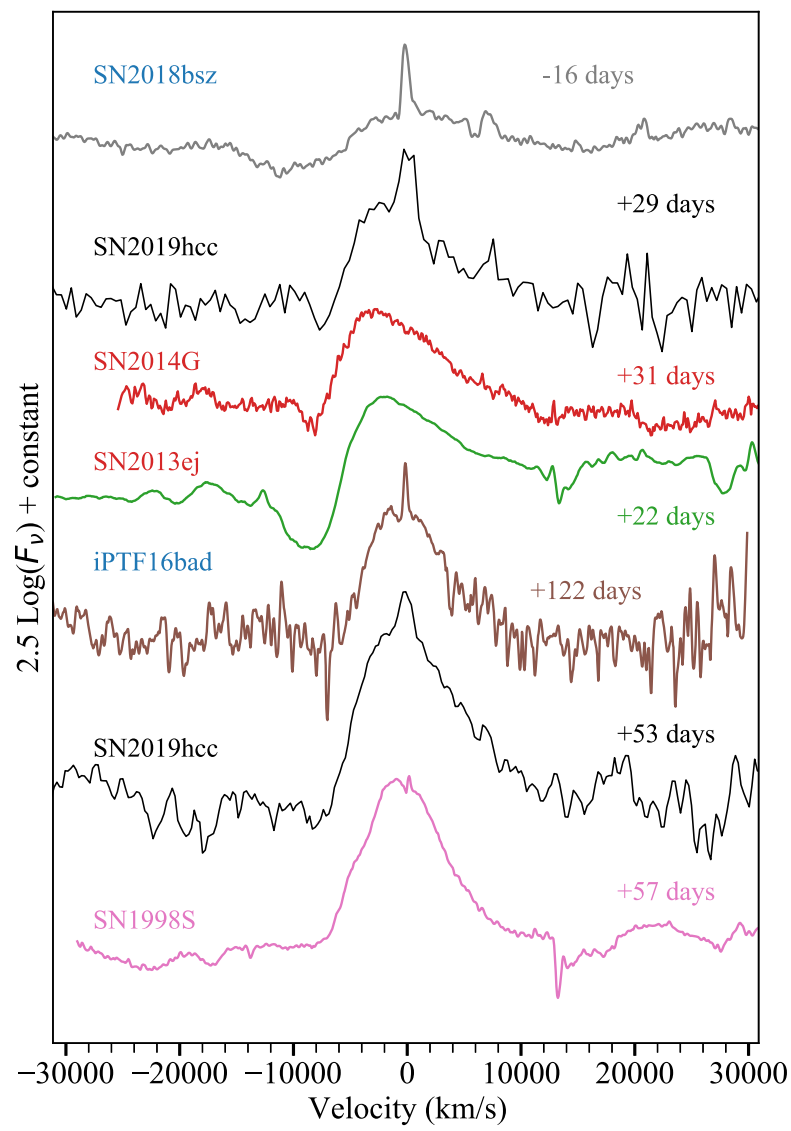

Figure 11. A comparison of the $\mathrm{H} \alpha$ profiles for a variety of moderately luminous SNe II and SLSNe I. The wavelength is in the rest frame. Text in red refers to Type II, while in blue to SLSNe I. The velocity is with respect to $\mathrm{H} \alpha$.

comparison as it is a SLSN I displaying a w-shaped profile at early times and $\mathrm{H} \alpha$ at late times. SN $2019 \mathrm{hcc}$ has a good match with some features, e.g. Balmer lines, however there are some discrepancies in the comparison, such as the lack of a P-Cygni profile for $\mathrm{H} \alpha$ in iPTF16bad. The Fe II lines at approximately $5000 \AA$ are also not observable in the spectrum of the SLSN I. If the $\mathrm{H} \alpha$ in SN 2019hcc was a consequence of interaction similar to iPTF16bad, we would expect other signs of interaction. These could be undulations or a second peak in the light curve (e.g. Nicholl et al. 2016; Inserra et al. 2017), but the SN 2019hcc light curve appears to be that of a typical SN IIL (see Section 5). Additionally, the relatively earlier appearance of the $\mathrm{H} \alpha$ emission in $\mathrm{SN} 2019$ hcc would require a much closer $\mathrm{H}$ shell than for iPTF16bad.

Fig. 10 also displays a comparison to moderately luminous SNe II SN 2013ej, SN 2014G, and SN 1998S. SN 1998S did not have a spectrum available at the $+29 \mathrm{~d}$ epoch, so is shown at the nearest later epoch, with SN 2019hcc at $+53 \mathrm{~d}$ for comparison. The spectra do not significantly evolve in this time frame. There are strong similarities between spectral features at the epoch of comparison, with good matches of $\mathrm{H} \alpha$ and Fe II features. The comparison would strengthen that SN 2019hcc is a Type II.

Fig. 11 shows a closer look at the $\mathrm{H} \alpha$ profiles for the previous spectra, and additionally SN 2018bsz, a SLSN I. In SLSNe I, carbon lines produced in the $\mathrm{H} \alpha$ region could be mistaken for hydrogen, such as in the case of SN 2018bsz, which displays C II $\lambda 6580$ line in the $\mathrm{H} \alpha$ region (Anderson et al. 2018a). SN 2018bsz does also show hydrogen but it is not observed at the phase being considered here. 
However, if $\mathrm{C}$ II is present in a spectrum, we should observe it at $\lambda 7234$ and $\lambda 5890$ (Anderson et al. 2018a), lines which are not seen in SN 2019hcc, while $\mathrm{H} \beta$ can be seen at $\lambda 4861$. This strengthens the idea that is indeed $\mathrm{H} \alpha$ observed in SN 2019hcc as opposed to C II.

SN 2019hcc spectra show an emission redward of $\mathrm{H} \alpha$ at approximately $6720 \AA$ visible at +29 d. Fig. 11 shows that SN 2018bsz also contains the redward emission at approximately $6720 \AA$. Singh et al. (2019) identifies this as [S II] lines at 6717 and $6731 \AA$ from the parent $\mathrm{H}$ II region.

\subsection{Investigating signs of interaction in the photospheric spectra}

A multicomponent $\mathrm{H} \alpha$ profile which does not completely hide the absorption component hints to a degree of interaction. Here, the narrow component would belong to the unshocked wind, whilst the medium component to the shocked wind/ejecta. Another sign of interaction between the ejecta and the CSM could be a high velocity (HV) component in the Balmer lines (e.g. Inserra et al. 2013a; Gutiérrez et al. 2017a). The normal velocity originates from the receding photosphere, whilst the HV is generated further out where the CSM interaction may excite the hydrogen to cause a second, high-velocity absorption feature (e.g. Arcavi 2017). The size and shape of this feature could be related to the progenitor wind density (Chugai, Chevalier \& Utrobin 2007). A small absorption bluer than the $\mathrm{H} \alpha$ P-Cygni has been observed in several SNe II but its nature is not always linked to $\mathrm{H} \alpha$ (Gutiérrez et al. 2017a). Such a feature, named 'Cachito', has previously been attributed to HV features of hydrogen, or Si II $\lambda 6533$. These features were identified in Inserra et al. (2013a) for some moderately luminous SNe ii. Gutiérrez et al. (2017a) also found the 'Cachito' feature is consistent with Si II at early phases, and with hydrogen at later phases.

\subsubsection{High-velocity features}

In the top panel of Fig. 12, an absorption blueward of $\mathrm{H} \alpha$ can be seen in SN 2019hcc at +19 and $+29 \mathrm{~d}$ at around $6250 \AA$ A, however, after this epoch it becomes less clear. An absorption feature can also be seen in SN 2013ej, and arguably SN 2014G, as seen in Fig. 11. The presence of a potential $\mathrm{HV} \mathrm{H} \beta$ additional to the $\mathrm{H} \alpha$ at a similar velocity would strengthen the latter's status as a HV feature of hydrogen (e.g. Chugai et al. 2007; Gutiérrez et al. 2017a; Singh et al. 2019). The lower panel on Fig. 12 shows the $\mathrm{H} \beta$ profile for SN 2019hcc at the epochs where it is visible, and an absorption blueward of the P-Cygni could be identified. Gutiérrez et al. (2017a) found that 63 per cent of their sample of SNe II with $\mathrm{HV} \mathrm{H} \alpha$ in the plateau phase showed a $\mathrm{HV} \mathrm{H} \beta$ at the same velocity. Gutiérrez et al. (2017a) also reported that if the absorption is produced by Si II its velocity should be similar to those presented by other metal lines, such as Fe II $\lambda 5169$, a good estimator for the photospheric velocity (Hamuy et al. 2001).

The velocity of this possible $\mathrm{H} \alpha \mathrm{HV}$ absorption feature in SN 2019hcc was measured at +19 and +29 d, with respect to $\mathrm{H} \alpha$ and $\mathrm{Si}$ II at $\lambda 6355$. The Fe II lines were also measured for comparison. Fig. 13 displays the measured velocities in SN 2019hcc for various lines at different epochs in its evolution. The velocity was found by fitting a Gaussian to the absorption features and finding the minimum - after $+29 \mathrm{~d}$, this fitting was not successful, therefore there are only two points available. With reference to Fig. 13, it can be seen that the measured Si II velocity is close to the Fe II velocity at both epochs, suggesting that it is near the photospheric velocity. This would lend
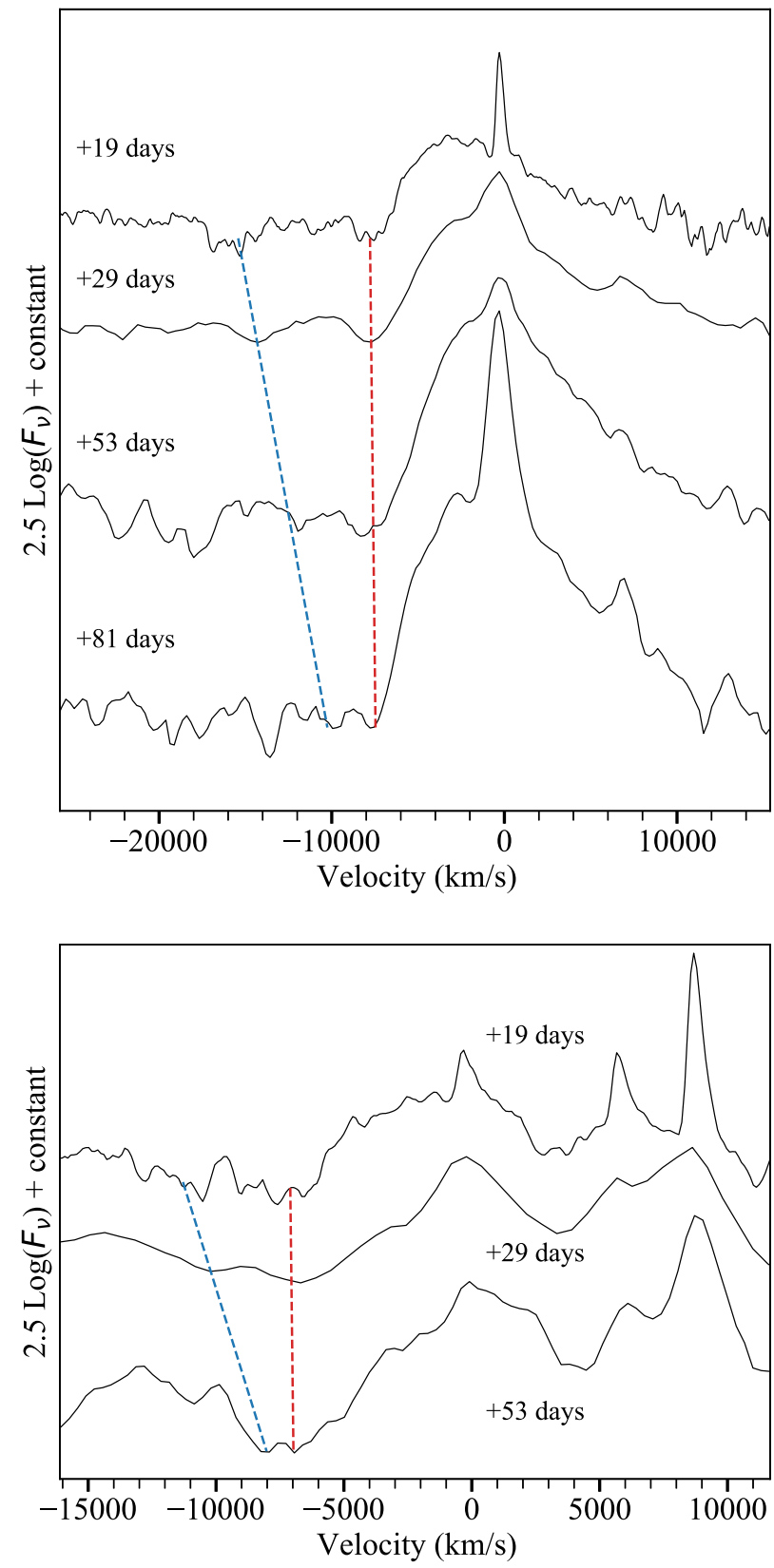

Figure 12. Top panel: the $\mathrm{H} \alpha$ profile evolution of SN 2019hcc, the spectra have been smoothed using a moving average. The velocity is with respect to $\mathrm{H} \alpha$. All spectra show a small feature blueward of $\mathrm{H} \alpha$ after smoothing, which could be a HV component indicating early CSM-ejecta interaction. The red dashed line tracks the $\mathrm{H} \alpha$ absorption, and the blue dashed line the possible $\mathrm{HV}$ component. Bottom panel: the same as the above panel but with respect to $\mathrm{H} \beta$. The velocity is with respect to $\mathrm{H} \beta$.

support to the feature being more likely associated with $\mathrm{Si}$ II. For the $\mathrm{HV}$ component, it would be expected the velocity of the $\mathrm{HV} \mathrm{H} \beta$ to match that of the $\mathrm{HV} \mathrm{H} \alpha$, and this is not what is found by our velocity analysis. Considering this information, the velocity measurements support that this feature is most likely associated with Si II.

Velocities were also measured for the lines of $\mathrm{H} \alpha, \mathrm{H} \beta$, and Fe II in SN 2014G and SN 1998S as shown in Fig. 13, and the Fe II velocities are similar to those of SN $2019 \mathrm{hcc}$ - although due to the scarcity of points for SN 2019hcc a meaningful comparison of the velocity evolution is difficult. Additionally, the average velocities of these lines 


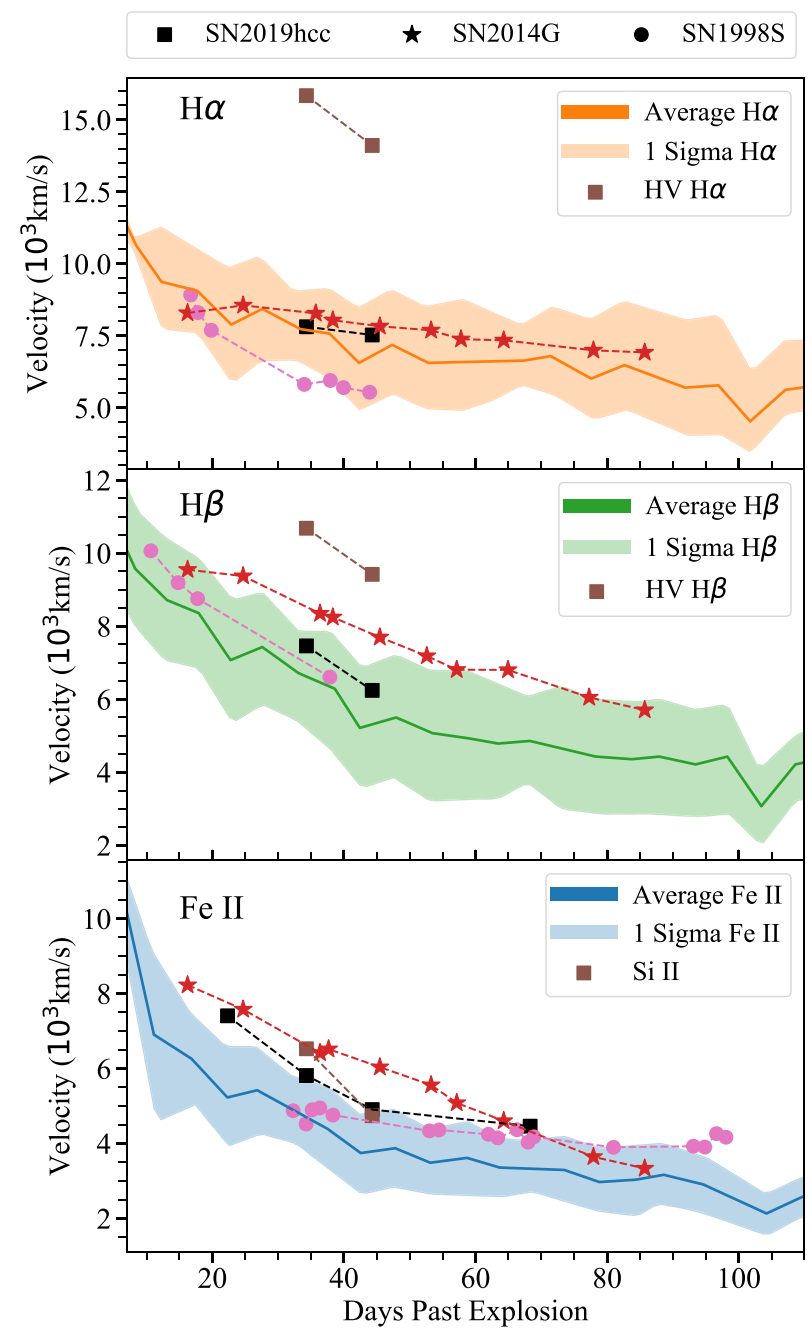

Figure 13. The velocity comparisons of different lines for SN $2019 \mathrm{hcc}$, over three different epochs, alongside the velocities of SN 2014G for Fe II, $\mathrm{H} \alpha$, and $\mathrm{H} \beta$. Velocities of SN 1998 S from Anupama, Sivarani \& Pandey (2001) and Terreran et al. (2016), SN 2014G from Terreran et al. (2016). The average velocities are found from 122 Type II SNe (Gutiérrez et al. 2017a), the figure reproduced from Dastidar et al. (2021) and reference therein, shown with a $1 \sigma$ error.

as measured by Gutiérrez et al. (2017a) for a sample of 122 SNe II are included in the plot, and show the velocities measured for SN 2014G, SN 2019hcc, and SN 1998S are roughly as expected for SNe II.

\subsubsection{Photospheric $\mathrm{H} \alpha$ profile}

Another sign of interaction in the spectra would be a multicomponent $\mathrm{H} \alpha$ profile with additional components to a simple P-Cygni profile. To investigate the possible presence of multicomponents, the profile of SN 2019hcc taken from its highest resolution spectrum at $+19 \mathrm{~d}$ was decomposed by means of Gaussian profiles. In a non-perturbed SN ejecta, the expected components would be both an absorption and an emission from the P-Cygni, as well as emission from the host galaxy. Any additional component could therefore suggest an ongoing ejecta-CSM interaction.

In Fig. 14, we display a composite Gaussian function. The $\mathrm{H} \alpha$ profile at $+19 \mathrm{~d}$ was chosen as it is the highest resolution spectrum of SN $2019 \mathrm{hcc}$, with a resolution of $6.0 \AA$. As can be seen, the

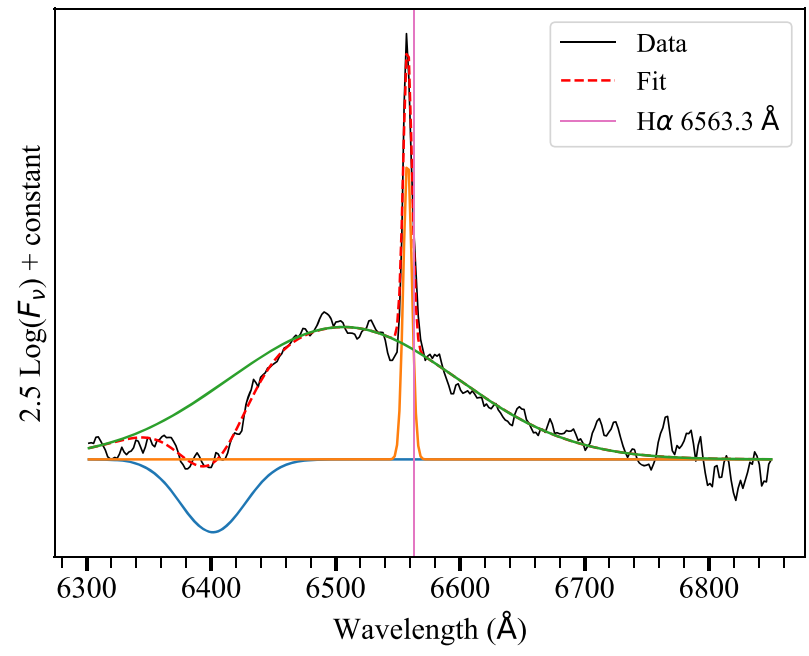

Figure 14. The $\mathrm{H} \alpha$ profile for $\mathrm{SN} 2019 \mathrm{hcc}$ at $+19 \mathrm{~d}$ past maximum with model profiles composed by several Gaussian profiles: a blueshifted $\mathrm{H} \alpha$ absorption (blue), the galaxy line (orange), a blueshifted $\mathrm{H} \alpha$ (green).

multicomponent function provides a good fit. The fit contains an absorption and emission component to reproduce the ejecta PCygni profile and a narrow emission component for the host galaxy. An additional broad Gaussian component could be due to CSM interaction, however, no additional component is required for the fit. The emission component was initially fitted with both a Gaussian and a Lorentzian fit, retrieving similar $\chi^{2}$ values. A Lorentzian profile is typically associated with scattering of photons in an optically thick CSM, and this requires a dense scattering medium (e.g. Reynolds et al. 2020). A better fit with a Lorentzian function indicates that broadening is due to electron scattering rather than expansion (e.g. Chatzopoulos et al. 2011; Taddia et al. 2013; Nicholl et al. 2020). However, as a Lorentzian is not a significantly better fit, this scenario is not supported.

P-Cygni theory predicts emission of hydrogen to peak at zero rest velocity $\lambda 6563.3$, however, observations reveal that emission peaks are often blueshifted (Anderson et al. 2014a). Anderson et al. (2014a) found that significant blueshifted velocities of $\mathrm{H} \alpha$ emission peaks are common and concluded that they are a fundamental feature of $\mathrm{SNe}$ spectra. This has been suggested to be due to the blocking of redshifted emission from the far side of the ejecta by an optically thick photosphere, due to a steep density profile within the ejecta (e.g. Reynolds et al. 2020). The fit allows for a blueshifted broad emission line as well as host galaxy emission at the rest wavelength. The Doppler shift from $\mathrm{H} \alpha$ in Fig. 14 is $2610 \pm 140 \mathrm{~km} \mathrm{~s}^{-1}$. Anderson et al. (2014a) found blueshifted emission velocities of the order of $2000 \mathrm{~km} \mathrm{~s}^{-1}$ therefore this result is consistent. Overall, the above analysis shows that a multicomponent profile is not necessary to reproduce the observed $\mathrm{H} \alpha$ profile and hence the spectra do not show any evidence of an ejecta-CSM interaction.

\section{THE EARLY ' $W$ ' SHAPED FEATURE: ELEMENTS CONTRIBUTION AND THEIR NATURE}

One of the most interesting features displayed by SN 2019hcc is its early ' $w$ ' shaped feature resembling that of SLSNe. Understanding its nature, composition, and the possibility that it is not a trademark of SLSNe I will have important consequences during the Vera C. Rubin and the Legacy Survey for Space and Time (LSST) era. LSST 


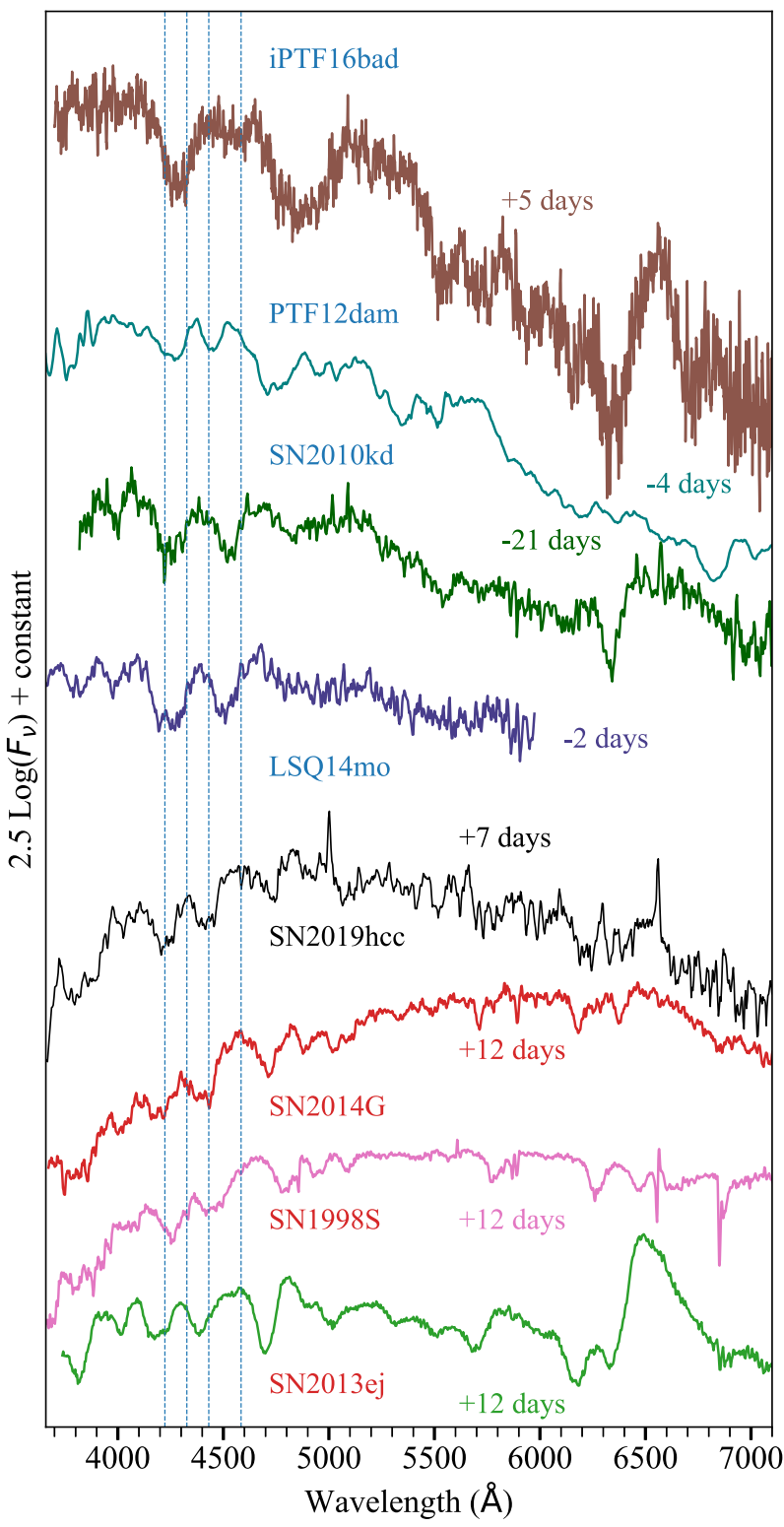

Figure 15. SN $2019 \mathrm{hcc}+7 \mathrm{~d}$ post-peak is compared to moderately luminous SNe II and SLSNe I. These spectra are displayed in terms of F $(v)$ to emphasize the absorption features, and the wavelength is in the rest frame. SN 2014G also appears to show a ' $w$ ' shaped profile at 4000-4400 $\AA$. The dashed lines correspond to the peaks and troughs of the O II line region in SN 2019hcc. Text in red represents Type II, text in blue SLSN.

will deliver hundreds of SLSNe (Inserra et al. 2021) and thousands of CC-SNe for which we might not have the luxury of multiple epoch spectroscopy.

Fig. 15 shows the OII features in the early spectrum for SN 2019hcc, together with the Type II SNe used for previous comparison, and the previous sample of SLSNe I. The approximate location of peaks and troughs of the SN 2019hcc O II lines is marked by dashed vertical lines for comparison. iPTF16bad (Yan et al. 2017) was chosen due to the late $\mathrm{H} \alpha$ emission, and SN 2010kd (Kumar et al. 2020) for the carbon emission which resembles $\mathrm{H} \alpha$. PTF12dam (Nicholl et al. 2013) was chosen for being a well-sampled SLSN I, and LSQ14mo (Chen et al. 2017b) for its similarity to SN 2019hcc with respect to the O II feature at a similar epoch. SN 2014G, amongst the SNe II, appears to have the strongest resemblance to SN 2019hcc, showing a similar pattern in the wavelength region around $4000 \AA$. A point to note is that SN 2019 hcc does not entirely match the $\mathrm{O}$ II feature in the SLSNe I - the redder absorption is blueshifted in comparison.

The features usually associated with O II are formed by many tens of overlapping lines (Anderson et al. 2018b; Gal-Yam 2019), and can be contaminated by carbon and metal lines, and also by the presence of well-developed Balmer lines, all of which mean the features cannot be uniquely identified as O II. Therefore, whilst SN 2019hcc, SN 2014G, and SN 1998S could be valid candidates to show O II features as the Balmer lines are less prominent, SN 2013ej is less likely as it shows a strong $\mathrm{H} \alpha$ profile suggesting the spectrum is dominated by $\mathrm{H} \beta$ at $\lambda 4861$ and $\mathrm{H} \gamma$ at $\lambda 4340$.

Gal-Yam (2019) tackled the challenge of line identification with comparison of absorption lines to lists of transitions drawn from the National Institute of Standards and Technology (NIST) database. $\mathrm{He}$ found that $\mathrm{O}$ II emission lines appear in the gaps between $\mathrm{O}$ II absorption, which corresponds to the two peaks - see Fig. 15 2nd and 4th dashed lines from the left. Anderson et al. (2018b) suggested that a change in the morphology of the spectrum in this wavelength region (between $\mathrm{SNe}$ ) may be produced through differences in ejecta density profiles or caused by overlapping lines such as Fe III.

Oxygen lines appear when oxygen is ionized by sufficiently high temperatures, 12000-15000 K (e.g. Inserra 2019). However, the presence of O II lines around 4000-4400 A might be a consequence of non-thermal excitation (Mazzali et al. 2016). This requires a power source in the CO core of massive stars (Mazzali et al. 2016). A lack of O II lines would be be the product of rapid cooling or lack of non-thermal sources of excitation (Quimby et al. 2018).

A non-thermal excitation could be in the form of strong X-ray flux from a magnetar, such as the injection of X-rays from an interaction between the SN ejecta and a magnetar wind (Maeda et al. 2007). Vurm \& Metzger (2021) modelled SLSNe powered by a relativistic wind from a central engine, such as a millisecond pulsar or magnetar, which inflates a nebula of relativistic electron/positron pairs and radiation behind the expanding supernova ejecta shell. These quickly radiate their energy via synchrotron and inverse Compton (IC) processes in a broad spectrum spanning the X-ray/gamma-ray band, a portion of which heats the ejecta and powers the supernova emission. This process will be most efficient at early times after the explosion, when the column density through the ejecta is at its highest. Non-thermal excitation could also be due to high-energy electrons produced by $\gamma$-rays from the radioactive decay of ${ }^{56} \mathrm{Ni}(\mathrm{Li}$, Hillier \& Dessart 2012), however, such a process would more likely be relevant at later times. It could also be produced by ejecta-CSM interaction (Nymark, Fransson \& Kozma 2006), with a CSM rich in oxygen producing the associated spectral features (Chatzopoulos \& Wheeler 2012). No SLSN I to date has shown narrow lines in its spectra (Nicholl et al. 2014; Inserra 2019), and interaction models are yet to reproduce the observed spectra. Nevertheless, the interaction model is still favoured to reproduce the light-curve evolution of some SLSNe I (e.g. Chatzopoulos et al. 2013).

Though supposed to be typical to SLSNe I (Branch \& Wheeler 2017), O II lines have already been seen in other SNe, such as SN Ibn OGLE-2012-SN-006 (Pastorello et al. 2015) and SN Ib SN 2008D (Soderberg et al. 2008, Modjaz et al. 2009). SN 2008D was a normal core-collapse SN with an associated X-Ray flash (e.g Li 2008), whereas OGLE-2012-SN-006 was interpreted as a corecollapse event powered by ejecta-CSM interaction (Pastorello et al. 2015). The presence of O II spectroscopic features here support the argument that ejecta-CSM interaction may be an important factor in maintaining the high levels of energy required to ionize oxygen (Pastorello et al. 2015). 


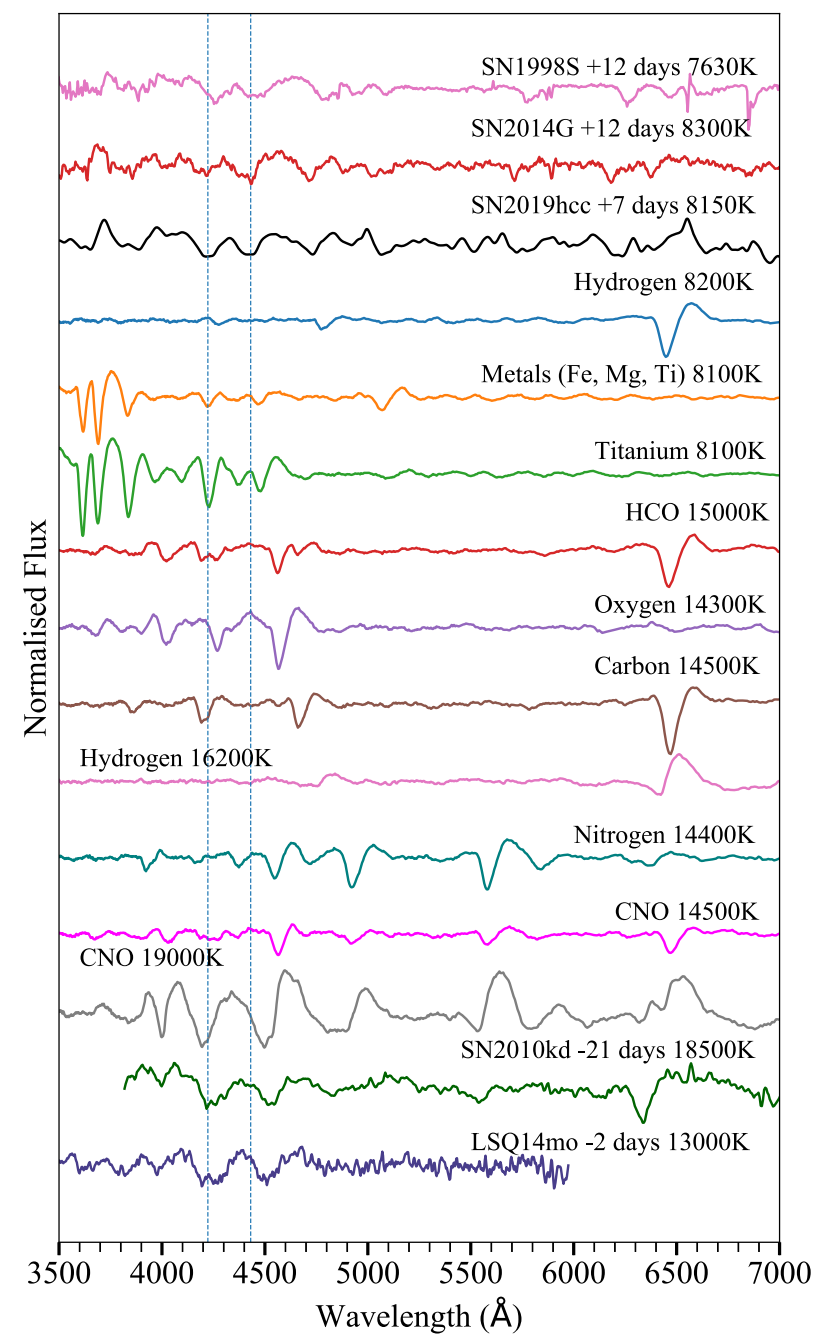

Figure 16. The output of the TARDIS modelling - spectra with various abundances and temperature. The vertical dashed lines mark the absorption lines for the SN 2019hcc 'w' feature.

\subsection{Spectral modelling}

Reproducing the ' $w$ ' shape of the first spectrum with spectral modelling could cast light on the conditions required to produce it. If the feature is reproduced by modelling oxygen at a higher temperature than the spectra which display this feature, it would suggest non-thermal excitation is necessary to produce this feature.

We used TARDIS (Kerzendorf \& Sim 2014), an open-source radiative transfer code for spectra modelling of $\mathrm{SNe}$, to model SN 2019hcc's first spectrum. The code uses Monte Carlo methods to obtain a self-consistent description of the plasma state and compute a synthetic spectrum. TARDIS was originally designed for Type Ia $\mathrm{SNe}$ and recently improved to be used for Type II spectra (Vogl et al. 2019), although the time varying profile of $\mathrm{H} \alpha$ remains difficult to reproduce. TARDIS assumes that the ejecta is in a symmetric and homologous expansion, and as such there is a direct correlation between time since explosion and the temperature at this time.

SN 2019hcc was modelled as having a uniform ejecta composition and the results are presented in Fig. 16. Model spectra were created with various abundances and temperatures and then normalized for comparison with SN 2019hcc. The temperatures were chosen to be around $8100 \mathrm{~K}$ (near the measured temperature of SN 2019hcc) or around $14000 \mathrm{~K}$ (closer to the SLSNe I used for comparison, see Fig. 7). Higher temperatures up to around $20000 \mathrm{~K}$ were also considered in order to investigate the effect of the temperature on the resulting spectra. The velocity was kept constant for all spectra, at $8000 \mathrm{~km} \mathrm{~s}^{-1}$ (start $6000 \mathrm{~km} \mathrm{~s}^{-1}$, stop $8000 \mathrm{~km} \mathrm{~s}^{-1}$ ), similar to the photospheric velocity measured by Fe II (see Fig. 13). Elements were investigated individually - with abundances of up to 100 per cent for one element. Starting from the approximate epoch and luminosity of SN 2019hcc, the spectra at approximately $8100 \mathrm{~K}$ were modelled by adjusting the input parameters until matching the temperature to that measured from the $+7 \mathrm{~d}$ spectrum for SN 2019hcc after Cardelli correction, as marked in the figure. The high temperature spectra around $15000 \mathrm{~K}$ were found by increasing the luminosity and decreasing the time since explosion in the model.

Modelling revealed that at the lower temperature of $8100 \mathrm{~K}$, carbon, oxygen, and helium are not sufficiently excited to show any lines therefore they have been omitted from the figure. However, metal ( $\mathrm{Fe}, \mathrm{Mg}$, and $\mathrm{Ti}$ ) and Balmer lines do show line profiles in this region which could have the potential to reproduce the absorption lines seen for SN 2019hcc. Hydrogen does not have largely significant absorption in this region compared to these metals. Also shown in Fig. 16 are elements at a higher temperature which is typical of SLSNe I at a similar phase to SN 2019hcc's first spectrum (see Fig. 7). These do not match well the overall spectrum of SN 2019 hcc but it can be noted that carbon, oxygen and nitrogen produce lines in the region of interest.

The bottom model spectrum of Fig. 16 shows that at approximately $19000 \mathrm{~K} \mathrm{a}$ 'w' feature can be produced with a CNO composition (with an even split of abundances). Note that nitrogen has a relatively small effect in comparison to carbon and oxygen in producing this shape. The 'w' feature for SN 2019hce is slightly shifted compared to the SLSNe I used for previous comparison - such a shift is evident in the red absorption but not the blue. A possible explanation for SN 2019hcc 'w' profile could be a combination of metals at a lower temperature $(8100 \mathrm{~K})$ and a non-thermally excited CNO layer. Considering that the temperatures of LSQ14mo and SN 2010kd are around $13000 \mathrm{~K}$ (at this temperature CNO does not show a ' $\mathrm{w}$ ' feature), this could confirm that these SLSNe I require non-thermal excitation to produce this feature.

The feature of SN 1998S looks different to SN 2019hcc - both lines of the ' $w$ '-feature have a different shape. The ' $w$ ' feature in SN 1998S is likely caused by titanium and a combination of other metals like Barium (Faran et al. 2014), which is also seen at redder wavelengths in SN 1998S but not in SN 2019hcc. Titanium does not look responsible for SN 2014G or SN 2019hcc as the ratios and shapes of the two profiles are different. The contribution from the combination of metals including Iron can be seen clearly in SN 2019hcc at $5169 \AA$; however, iron lines cannot account for the strong absorption in the ' $w$ ' feature region. Reproducing the strength of lines would appear to require $\mathrm{CNO}$ abundances at higher temperatures - for example oxygen and carbon at approximately $14000 \mathrm{~K}$ could account for the broader red wing of SN 2019hcc. A combination of CNO at higher temperatures than SN 2019hcc spectrum (i.e. $8100 \mathrm{~K}$ ) and metals at $8100 \mathrm{~K}$ could be causing the final feature. However, with the tested models it seems impossible to completely reproduce the ' $w$ ' feature. Nevertheless, it appears models at $T>14000 \mathrm{~K}$ are required to reproduce the strength of the absorption, suggesting a non-thermal excitation responsible for the CNO elements SN 2019hcc at $+7 \mathrm{~d}$.

Equivalent width (EW) ratios are measured in order to provide a more quantitative analysis of the feature. These are reported in Table 3 in the form of the EW of the blue line over the red one, as well as the 
Table 3. Equivalent widths (EW) and FWHM of the absorption of the blue line profile over the red of the ' $w$ ' feature.

\begin{tabular}{lccc}
\hline SN name & Type & EW (blue/red) & FWHM (blue/red) \\
\hline SN 2019hcc & SN IIL & $1.11 \pm 0.05$ & $1.06 \pm 0.03$ \\
SN 2014G & SN IIL & $0.77 \pm 0.03$ & $1.03 \pm 0.05$ \\
SN 1998S & SN IIn & $0.94 \pm 0.06$ & $0.77 \pm 0.04$ \\
SN 2010kd & SLSN I & $1.39 \pm 0.07$ & $1.24 \pm 0.02$ \\
LSQ14mo & SLSN I & $1.61 \pm 0.06$ & $1.29 \pm 0.04$ \\
\hline
\end{tabular}

same ratio for full width at half maximum (FWHM). Of the SNe II, only SN 2019hcc has an EW over 1. The SLSNe I in this table also have a ratio over 1 and are larger with respect to that of SN 2019hcc. In both cases the SLSNe I have a slightly higher FWHM than the $\mathrm{SNe}$ II although this is not statistically conclusive due to the small size of the sample. These ratios cannot offer anything conclusive as it suggests all these 'w' features are of a slightly different nature, and could possibly be affected by temperatures, abundances, nonthermal excitation, or the presence of other lines such as metal lines. Possibly SN 2014G could also be non-thermally excited, or have different metal contributions, though its nature looks different to the other $\mathrm{SNe}$ as it is the only spectrum with a significantly stronger red line than blue.

In summary, at temperatures of approximately $19000 \mathrm{~K} \mathrm{CNO}$ could reproduce the ' $w$ ' feature. Some absorption in this region at a temperature of $8100 \mathrm{~K}$ could be caused by metal lines e.g. titanium, however, this cannot entirely account for the ' $w$ ' feature in SN 2019hcc spectrum. Metals would also produce stronger lines at bluer wavelengths (3500-4000 A) which are not seen in SN 2019hcc, though these could be obscured by yet more lines in this region. For thermally exciting $\mathrm{CNO}$ much higher temperatures are needed than that observed for SN 2019hcc therefore non-thermal excitation may be required to produce such features in SN 2019hcc. This appears to also be the case for LSQ14mo and SN2010kd, which show the feature despite LSQ14mo being almost $6000 \mathrm{~K}$ short of the required excitation temperature.

He I can also be non-thermally excited, however, this excitation usually comes from CSM interaction at the outer boundary of the ejecta (e.g. Chevalier \& Fransson 1994), whereas for the non-thermal excitation of $\mathrm{O}$ II in this scenario the exciting X-ray photons would originate from the central engine. The ejecta helium region would be further away than the oxygen region for these central high-energy photons which, in our proposed scenario, would explain the absence of He I in the first spectrum of SN 2019hcc. Additionally, though the abundance of oxygen in the progenitor is relatively low compared to other elements such as hydrogen, the first spectrum is relatively featureless so $\mathrm{O}$ II is not competing with other lines in this region.

Hence, the next question to address is what could cause the nonthermal excitation of such $\mathrm{CNO}$ lines.

\subsection{Ejecta-CSM interaction scenario}

The presence of $\mathrm{O}$ II lines could be the consequence of ejecta-CSM interaction (e.g. Pastorello et al. 2015). Mazzali et al. (2016) suggested that X-rays would be required for the non-thermal excitation of $\mathrm{O}$ II lines, and these X-rays could originate from interaction (Nymark et al. 2006). However, Chevalier \& Fransson (1994) suggested that in ejecta-CSM interaction with an SN density profile consistent with that of an RSG progenitor, as with the majority of Type II, the photons produced would be primarily in the UV-range, thus not providing sufficient non-thermal excitation to ionize the oxygen.
There are no distinctive narrow emission lines in the spectrum of SN 2019hcc, nor is there any unusual behaviour in the light curve such as multiple peaks or undulations which would suggest collision with a shell (e.g. Nicholl et al. 2016; Inserra et al. 2017). A possible $\mathrm{HV}$ component of $\mathrm{H} \alpha$ blueward of the main emission could be indicative of early weak/moderate CSM-ejecta interaction - as this interaction may excite the hydrogen to cause a second, high-velocity absorption feature (e.g. Arcavi 2017, [). However, our results on the $\mathrm{HV} \mathrm{H} \alpha$ analysis reported in Section 6.2.1 suggest that the presence of a $\mathrm{HV} \mathrm{H} \alpha$ is unlikely with the absorption blueward than $\mathrm{H} \alpha$ plausibly associated with $\mathrm{Si}$ II. The overall $\mathrm{H} \alpha$ profile was also analysed and decomposed in multiple components investigating the nature of the profile. However, it was found that no additional components are required to reproduce the shape aside from the expected ejecta PCygni and the narrow $\mathrm{H} \alpha$ line from the host galaxy. Therefore, CSM-ejecta interaction is not a viable source for generating highenergy photons capable of non-thermally excited the $\mathrm{O}$ II lines in SN 2019hcc.

\subsection{Magnetar scenario}

A magnetar could produce the non-thermal excitation required to ionize oxygen and produce the OII features (e.g. Mazzali et al. 2016). Dessart et al. (2012) suggested the magnetar's extra energy heats material and thermally excites the gas. Alternatively, Gilkis, Soker \& Papish (2016) and Soker \& Gilkis (2017) suggested that magnetar-driven SLSNe are powered not by the neutrino-driven mechanism but a jet feedback mechanism from jets launched at magnetar birth. These high-energy jets could potentially provide the energy to drive O II excitation at early times, and have been used to link magnetars to Gamma Rays Bursts (GRBs; Wheeler et al. 2000). The generation of a non-relativistic jet during the early supernova phase is a consequence in both the core-collapse and magnetar models of GRBs (Burrows et al. 2007).

Kasen \& Bildsten (2010) suggested that a magnetar birth is likely to happen in a few per cent of all core-collapse supernovae, and may naturally explain some of the brightest events seen. Orellana, Bersten \& Moriya (2018) found that magnetar-powered models can actually generate a diversity of hydrogen-rich $\mathrm{SNe}$, both ordinary and brighter ones. Through their modelling, it was found that the observational appearance of SNe II powered by magnetars can be extremely varied and can also mimic those of normal SNe IIP. Magnetars are thought to form by fast rotation in the collapsing Iron core (Duncan \& Thompson 1992). It is suggested that magnetars are preferentially formed in the most massive stars collapsing to a neutron star - with a progenitor mass in excess of $40 \mathrm{M}_{\odot}$ (Davies et al. 2009). However, it has also been suggested that magnetars do not require massive progenitors to form - alternatives could be a 'fossil-field' model, where a seed B-field is inherited from the natal molecular cloud (Davies et al. 2009) or an interacting binary system which causes spin-up in the collapsing CO-core (Cantiello et al. 2007).

Chen et al. (2017a) found an apparent correlation between magnetar spin-down period and host metallicity from a sample of 19 SLSNe I, indicating that faster-rotating magnetars reside in more metal-poor environments. Such a correlation could be a consequence of several factors - Martayan et al. (2007) found that massive stars rotate more rapidly at lower metallicity $\left(0.2 \mathrm{Z} / \mathrm{Z}_{\odot}\right)$ than solar, whilst Mokiem et al. (2007) found in low-metallicity environments massloss of rotating stars is reduced. However, the spin periods of lowmetallicity stars and neutron stars would also very likely be affected by other parameters. Generally, the greater the spin period, the greater the peak luminosity (Kasen \& Bildsten 2010; Inserra et al. 2013b), 


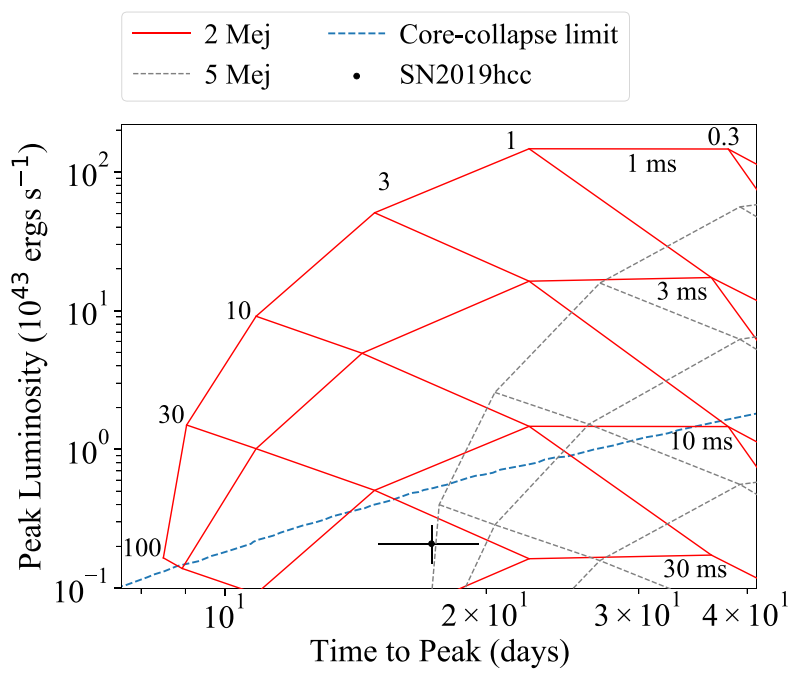

Figure 17. The effect of ejecta mass on a magnetar model grid (see text for further information about the grid limits and model used) - with an opacity of $0.34 \mathrm{~g} \mathrm{~cm}^{-3}$ suited for a H-rich ejecta. The grid markers are for Pms in $\mathrm{ms}$ and $\mathrm{B} 14$ in $\mathrm{B} / 10^{14} \mathrm{G}$.

therefore a high-metallicity host environment could be correlated with low-luminosity explosions powered or affected by a magnetar.

From the equations in Kasen \& Bildsten (2010), a grid of B14 $\left(\mathrm{B} / 10^{14} \mathrm{G}\right)$ and Pms (the spin period in $\mathrm{ms}$ ) of a magnetar as a function of the peak luminosity and rise time was produced, using the code presented in Inserra et al. (2013b). Multiple grids were created by varying the ejecta mass in the model, in order to investigate its effect. Fig. 17 shows an ejecta mass of $2 \mathrm{M}_{\odot}$ versus $5 \mathrm{M}_{\odot}$. These ejecta masses were chosen based on the bolometric lightcurve fitting of SN 2019hcc (using the code of Inserra et al. 2013b) and that of SN 2014G which is one of the other potential Type II showing the ' $w$ '-shaped feature. We retrieved an ejecta mass of approx. 2.3 and $5.0 \mathrm{M}_{\odot}$, respectively. The fitting was focused on matching the rise time and peak magnitude rather than attempting to accurately reproduce the entire shape of the light curve including the tail, as this is also affected by other factors such as ${ }^{56} \mathrm{Ni}$ or CSM interaction. The range of values in the grid are based on the fact that the neutron stars cannot spin faster than $1 \mathrm{~ms}$ without breaking up and that spin periods $<30 \mathrm{~ms}$ can substantially modify the thermal evolution of the supernova (Kasen \& Bildsten 2010), while $B$ values are those retrieved from galactic magnetars $\approx 10^{14}-10^{15} \mathrm{G}$ (Woods \& Thompson 2006). This figure shows that increasing the ejecta mass, but preserving B14 and Pms, would result in a longer rise time with the luminosity not as significantly affected. SN 2019hcc's location in this parameter space (see Fig. 17) shows that a lower luminosity supernova (i.e. not a SLSN) could be produced by a high magnetic field and a relatively lower spin. The blue-dashed line represents the core-collapse limit for peak luminosity versus rise time (Inserra 2019). Sukhbold \& Thompson (2017) also presented a proof-of-concept model of a magnetar mechanism producing Type IIP light-curve properties for a range of initial spin periods and equivalent dipole magnetic field strengths, and found for a $\mathrm{SNe}$ of peak bolometric luminosity of $\sim 42.5$, approximately that of SN 2019hcc, one would expect a Pms of $2 \mathrm{~ms}$ and a B14 of 100 - this agrees very well with the $5 \mathrm{M}_{\odot}$ model in Fig. 17 .

This modelling suggests it is possible to have a magnetar formed as a remnant without injecting further substantial energy to the supernova event leading to superluminous brightness. This could provide sufficient non-thermal contribution to excite the $\mathrm{O}$ II lines which appear in the early spectra. The sub-solar metallicity found in Section 2 would not provide support for the tentative hypothesis of a correlation between host environment metallicity and magnetar luminosity, as the metallicity is similar to that of the the typical low metallicity environments of SLSNe I, whilst the luminosity is typically lower than that of SLSNe I.

\section{CONCLUSIONS}

The first spectrum of SN 2019hcc appears relatively featureless aside from a 'w' feature around $4000 \AA$, characteristic of O II lines typical of SLSNe I. The redder absorption appears to be relatively blueshifted with respect to SLSNe I. The spectra show a clear $\mathrm{H} \alpha$ profile from $+19 \mathrm{~d}$, as well as spectral similarity to various literature SNe II, and the bolometric light-curve evolution is that of a SNe IIL. The host metallicity was sub-solar, a value lower than the typical Type II SNe (Gutiérrez et al. 2018). The temperature and colour evolution were typical of a Type II.

Such a 'w'-shaped feature (usually and historically) attributed to $\mathrm{O}$ II has never been identified and analysed in SNe II as such and only recognized in SN 2014G thanks to the analysis reported in this paper. Modelling of this 'w' feature using TARDIS (Kerzendorf $\&$ Sim 2014) suggested it could be produced by the excitation of $\mathrm{CNO}$ at a temperature of $19000 \mathrm{~K}$, which is more than twice that measured from the spectrum, suggesting these lines would therefore be non-thermally excited. Another result of the modelling was that absorptions at these wavelengths could also be the result of metal lines at $8100 \mathrm{~K}$, a temperature in agreement with that measured. In SLSNe I these lines have been suggested as excited by X-rays produced by a magnetar, or alternatively CSM-ejecta interaction. As there is a lack of any sign of interaction both in the light curve and spectra, aside from a tentative HV component, and potential interaction at late epochs, the CSM-ejecta interaction at early time is disfavoured. We built a model grid, following the work of Kasen \& Bildsten (2010) and using the code by Inserra et al. (2013b), and found that a magnetar could be formed as a remnant in a Type II. This would require that the magnetar does not provide enough additional energy to the supernova event to power up the light curve to superluminous luminosities. The magnetar remnant could therefore non-thermally excite the oxygen whilst not having a significant contribution to the light-curve evolution. Therefore, combining such results with those of the spectral modelling, we conclude that the ' $w$ ' feature seen in SN 2019hcc's first spectrum could be due to a combination of non-thermally excited CNO and thermally excited metal lines.

The object here presented could then bridge the gap between SLSNe I and normal luminosity core-collapse supernovae, as well as reveal more about magnetar formation requirements and mechanisms. Our analysis also shows that a magnetar is a viable remnant of a Type II supernova explosion, the effects on which could be observed in the form of an early ' $w$ '-shaped profile around 4000-4400 $\AA$. This would suggest that such lines are not exclusive to SLSNe I and cannot be used as a sole feature to classify those extreme transients.

\section{ACKNOWLEDGEMENTS}

Based on observations collected at the European Organisation for Astronomical Research in the Southern Hemisphere, Chile, as part of ePESSTO/ePESSTO+ (the extended Public ESO Spectroscopic Survey for Transient Objects Survey). ePESSTO+ observations were obtained under ESO program ID 1103.D-0328 (PI: Inserra).

EP would like to thank Stuart Sim for useful discussion on the working of TARDIS. This research made use of TARDIS, a community- 
developed software package for spectral synthesis in supernovae (Kerzendorf \& Sim 2014; Kerzendorf et al. 2019). The development of TARDIS received support from the Google Summer of Code initiative and from the European Space Agency's (ESA) Summer of Code in Space program. TARDIS makes extensive use of Astropy and PyNE.

TWC acknowledges the funding provided by the Alexander von Humboldt Foundation and the EU Funding under Marie SkłodowskaCurie grant H2020-MSCA-IF-2018-842471. TMB was funded by the Comisión Nacional de Investigación Científica y Tecnológica (CONICYT) PFCHA / DOCTORADOBECAS CHILE/2017-72180113. MG is supported by the Polish Narodowe Centrum Nauki (NCN) MAESTRO grant 2014/14/A/ST9/00121. MN is supported by a Royal Astronomical Society Research Fellowship. ACK: LG was funded by the European Union's Horizon 2020 research and innovation programme under the Marie Skłodowska-Curie grant agreement No. 839090. This work has been partially supported by the Spanish grant PGC2018-095317-B-C21 within the European Funds for Regional Development (FEDER). GL is supported by a research grant (19054) from VILLUM FONDEN

This work makes use of observations from the Las Cumbres Observatory (LCO) global telescope network. The LCO team is supported by National Science Foundation (NSF) grants AST1911225, AST-1911151, and NASA grant 80NSSC19K1639. This paper is also based on observations made with Swift (UVOT) and the Liverpool Telescope (LT). The Liverpool Telescope is operated on the island of La Palma by Liverpool John Moores University in the Spanish Observatorio del Roque de los Muchachos of the Instituto de Astrofisica de Canarias with financial support from the UK Science and Technology Facilities Council (STFC). LCO data have been obtained via Optical Infrared Co-ordination Network for Astronomy (OPTICON) proposals (IDs: SUPA2020B-002 OPTICON 20B/003 and SUPA2019B-007 OPTICON 19B-009). The OPTICON project has received funding from the European Union's Horizon 2020 research and innovation programme under grant agreement No 730890. This work has made use of data from the Asteroid Terrestrialimpact Last Alert System (ATLAS) project. The ATLAS project is primarily funded to search for near earth asteroids through NASA grants NN12AR55G, 80NSSC18K0284, and 80NSSC18K1575; byproducts of the Near-Earth Object (NEO) search include images and catalogs from the survey area. This work was partially funded by Kepler/K2 grant J1944/80NSSC19K0112 and HST GO-15889, and STFC grants ST/T000198/1 and ST/S006109/1. The ATLAS science products have been made possible through the contributions of the University of Hawaii Institute for Astronomy, the Queen's University Belfast, the Space Telescope Science Institute, the South African Astronomical Observatory, and The Millennium Institute of Astrophysics (MAS), Chile. This work has made use of data from the European Space Agency (ESA) mission Gaia (https: //www.cosmos.esa.int/gaia), processed by the Gaia Data Processing and Analysis Consortium (DPAC, https://www.cosmos.esa.int/web /gaia/dpac/consortium). Funding for the DPAC has been provided by national institutions, in particular the institutions participating in the Gaia Multilateral Agreement. Based on observations obtained at the Southern Astrophysical Research (SOAR) telescope, which is a joint project of the Ministério da Ciência, Tecnologia e Inovações (MCTI/LNA) do Brasil, the US National Science Foundation's NOIRLab, the University of North Carolina at Chapel Hill (UNC), and Michigan State University (MSU). This work has made use of data from the Gamma-ray Burst Optical/Near-infrared Detector (GROND) instrument at the 2.2 MPE telescope at La Silla, Chile. Part of the funding for GROND (both hardware as well as personnel) was generously granted from the Leibniz-Prize to Prof. G. Hasinger (Deutsche Forschungsgemeinschaft (DFG) grant HA 1850/28-1).
GROND data were obtained under European Southern Observatory (ESO) programme ID 0103.A-9099. This research has made use of the NASA/IPAC Extragalactic Database (NED), which is funded by the National Aeronautics and Space Administration and operated by the California Institute of Technology.

This research made use of Photutils, an Astropy package for detection and photometry of astronomical sources (Bradley et al. 2020). Based on data products from observations made with ESO Telescopes at the La Silla or Paranal Observatories under ESO programme ID 179.A-2010.

IRAF is distributed by the National Optical Astronomy Observatories, which is operated by the Association of Universities for Research in Astronomy, Inc. (AURA) under cooperative agreement with the National Science Foundation. This research made use of NUMPY (Harris et al. 2020), MATPLOTLIB (Hunter 2007), and ASTROPY (Astropy Collaboration 2013, 2018).

\section{DATA AVAILABILITY}

The data underlying this article are available in the article and in its online supplementary material. The photometry code is available at the following GitHub account https://github.com/eparrag1/Photom etry.

\section{REFERENCES}

Anderson J. P. et al., 2014a, MNRAS, 441, 671

Anderson J. P. et al., 2014b, ApJ, 786, 67

Anderson J. P. et al., 2016, A\&A, 589, A110

Anderson J. P. et al., 2018a, A\&A, 620, A67

Anderson J. P. et al., 2018b, A\&A, 620, A67

Anderson J. P., 2019, A\&A, 628, A7

Andrews J. E. et al., 2019, ApJ, 885, 43

Angus C. R. et al., 2019, MNRAS, 487, 2215

Anupama G. C., Sivarani T., Pandey G., 2001, A\&A, 367, 506

Arcavi I., 2017, Hydrogen-Rich Core-Collapse Supernovae. Springer International Publishing, Basel, Switzerland, p. 239

Asplund M., Grevesse N., Sauval A. J., Scott P., 2009, ARA\&A, 47, 481

Astropy Collaboration, 2013, A\&A, 558, A33

Astropy Collaboration, 2018, AJ, 156, 123

Barbon R., Ciatti F., Rosino L., 1979, A\&A, 72, 287

Bazin G. et al., 2009, A\&A, 499, 653

Benetti S. et al., 2016, MNRAS, 456, 3296

Bianco F. B., Modjaz M., Oh S. M., Fierroz D., Liu Y. Q., Kewley L., Graur O., 2016, Astronomy and Computing, 16, 54

Bose S. et al., 2015, ApJ, 806, 160

Bradley L. et al., 2020, astropy/photutils: 1.0.0. Accessed on 29th July 2021, Available at: https://doi.org/10.5281/zenodo.4044744

Branch D., Falk S. W., McCall M. L., Rybski P., Uomoto A. K., Wills B. J., 1981, ApJ, 244, 780

Branch D., Wheeler J. C., 2017, Supernova Explosions. Springer-Verlag GmbH, Heidelberg, Germany

Brown T. M. et al., 2011, American Astronomical Society Meeting Abstracts, Vol. 218, The Bulletin of the American Astronomical Society, p. 132.02

Burrows A., Dessart L., Livne E., Ott C. D., Murphy J., 2007, ApJ, 664, 416 Buzzoni B. et al., 1984, The Messenger, 38, 9

Caffau E., Ludwig H. G., Steffen M., Freytag B., Bonifacio P., 2011, Sol. Phys., 268, 255

Calzetti D., Armus L., Bohlin R. C., Kinney A. L., Koornneef J., StorchiBergmann T., 2000, ApJ, 533, 682

Cantiello M., Yoon S. C., Langer N., Livio M., 2007, A\&A, 465, L29

Cardelli J. A., Clayton G. C., Mathis J. S., 1989, ApJ, 345, 245

Chabrier G., 2003, PASP, 115, 763

Chambers K. C. et al., 2016, preprint (arXiv:1612.05560)

Chatzopoulos E. et al., 2011, ApJ, 729, 143

Chatzopoulos E., Wheeler J. C., 2012, ApJ, 760, 154 
Chatzopoulos E., Wheeler J. C., Vinko J., Horvath Z. L., Nagy A., 2013, ApJ, 773,76

Chen T. W. et al., 2017b, A\&A, 602, A9

Chen T. W. et al., 2018, ApJ, 867, L31

Chen T.-W. et al., 2017c, ApJ, 849, L4

Chen T.-W., Smartt S. J., Yates R. M., Nicholl M., Krühler T., Schady P., Dennefeld M., Inserra C., 2017a, MNRAS, 470, 3566

Chevalier R. A., Fransson C., 1994, ApJ, 420, 268

Chugai N. N., Chevalier R. A., Utrobin V. P., 2007, ApJ, 662, 1136

Clemens J. C., Crain J. A., Anderson R., 2004, in Moorwood A. F. M., Iye M., eds, Proc. SPIE Conf. Ser. Vol. 5492, Ground-based Instrumentation for Astronomy. SPIE, Bellingham, p. 331

Conroy C., Gunn J. E., White M., 2009, ApJ, 699, 486

Cutri R. M. et al. 2013, Explanatory Supplement to the AllWISE Data Release Products, Explanatory Supplement to the AllWISE Data Release Products. Accessed on 29th July 2021, Available at: https://wise2.ipac.c altech.edu/docs/release/allwise/expsup/index.html

Dastidar R., et al., 2021, MNRAS, 504, 1009

Davies B., Figer D. F., Kudritzki R.-P., Trombley C., Kouveliotou C., Wachter S., 2009, ApJ, 707, 844

Davis S. et al., 2019, ApJ, 887, 4

de Jaeger T. et al., 2018, MNRAS, 476, 4592

Delgado A., Harrison D., Hodgkin S., Leeuwen M. V., Rixon G., Yoldas A., 2019, Transient Name Server Discovery Report. Vol. 957, Transient Name Server, p. 1

Dessart L. et al., 2014, MNRAS, 440, 1856

Dessart L., Hillier D. J., 2020, A\&A, 642, A33

Dessart L., Hillier D. J., Waldman R., Livne E., Blondin S., 2012, MNRAS, 426, L76

Duncan R. C., Thompson C., 1992, ApJ, 392, L9

Faran T. et al., 2014, MNRAS, 445, 554

Fassia A. et al., 2000, MNRAS, 318, 1093

Fassia A. et al., 2001, MNRAS, 325, 907

Filippenko A. V., 1988, AJ, 96, 1941

Filippenko A. V., Matheson T., Ho L. C., 1993, ApJ, 415, L103

Foreman-Mackey D., Sick J., Johnson B., 2014, Python-Fsps: Python Bindings To Fsps (V0.1.1). Accessed on 29th Juldy 2021, Available at: https://ui.adsabs.harvard.edu/abs/2014zndo.....12157F/abstract

Fransson C., Chevalier R. A., 1989, ApJ, 343, 323

Frohmaier C. et al., 2019, Transient Name Server AstroNote, 27, 1

Frohmaier C. et al., 2021, MNRAS, 500, 5142

Gaia Collaboration, 2016, A\&A, 595, A1

Gal-Yam A., 2012, Science, 337, 927

Gal-Yam A., 2019, ApJ, 882, 102

Gal-Yam A., Yaron O. 2012, Publications of the Astronomical Society of the Pacific, 124, 668

Galbany L. et al., 2016, AJ, 151, 33

Galbany L. et al., 2018, ApJ, 855, 107

Gilkis A., Soker N., Papish O., 2016, ApJ, 826, 178

González-Gaitán S. et al., 2015, MNRAS, 451, 2212

Greiner J. et al., 2008, PASP, 120, 405

Guillochon J., Parrent J., Kelley L. Z., Margutti R., 2017, ApJ, 835, 64

Gutiérrez C. P. et al., 2017a, ApJ, 850, 89

Gutiérrez C. P. et al., 2017b, ApJ, 850, 90

Gutiérrez C. P. et al., 2018, MNRAS, 479, 3232

Gutiérrez C. P. et al., 2020, MNRAS, 499, 974

Hamuy M. et al., 2001, ApJ, 558, 615

Harris C. R. et al., 2020, Nature, 585, 357

Huang F. et al., 2015, ApJ, 807, 59

Hunter J. D., 2007, Comput. Sci. Eng., 9, 90

Inserra C. et al., 2013a, A\&A, 555, A142

Inserra C. et al., 2013b, ApJ, 770, 128

Inserra C. et al., 2017, MNRAS, 468, 4642

Inserra C., 2019, Nat. Astron., 3, 697

Inserra C., et al. 2021, MNRAS, 504, 2535

Jerkstrand A., Fransson C., Maguire K., Smartt S., Ergon M., Spyromilio J., 2012, A\&A, 546, A28

Johnson B. D., Leja J. L., Conroy C., Speagle J. S., 2019, Astrophysics Source Code Library, record ascl:1905.025
Kasen D., Bildsten L., 2010, ApJ, 717, 245

Kerzendorf W. E., Sim S. A., 2014, MNRAS, 440, 387

Kerzendorf W. et al., 2019, tardis-sn/tardis: Tardis v3.0 alpha2. Accessed on 29th July 2021, Available at: https://zenodo.org/record/2590539\#.YQUT xEDhWUk

Kewley L. J., Ellison S. L., 2008, ApJ, 681, 1183

Kozma C., Fransson C., 1998, ApJ, 497, 431

Krühler T. et al., 2008, ApJ, 685, 376

Kumar A. et al., 2020, ApJ, 892, 28

Lang D., 2014, AJ, 147, 108

Leja J., Johnson B. D., Conroy C., van Dokkum P. G., Byler N., 2017, ApJ, 837,170

Leloudas G. et al., 2015a, MNRAS, 449, 917

Leloudas G. et al., 2015b, ApJ, 815, L10

Li C., Hillier D. J., Dessart L., 2012, MNRAS, 426, 1671

Li L.-X., 2008, MNRAS, 388, 603

Lunnan R. et al., 2014, ApJ, 787, 138

Maeda K. et al., 2007, ApJ, 666, 1069

Mainzer A. et al., 2014, ApJ, 792, 30

Maiolino R. et al., 2008, in Funes J. G., Corsini E. M., eds, ASP Conf. Ser Vol. 396, Formation and Evolution of Galaxy Disks. Astron. Soc. Pac., San Francisco, p. 409

Martayan C., Frémat Y., Hubert A. M., Floquet M., Zorec J., Neiner C., 2007, A\&A, 462, 683

Martin D. C. et al., 2005, ApJ, 619, L1

Mazzali P. A., Sullivan M., Pian E., Greiner J., Kann D. A., 2016, MNRAS, 458,3455

McCrum M. et al., 2015, MNRAS, 448, 1206

McGaugh S. S., 1991, ApJ, 380, 140

McMahon R. G., Banerji M., Gonzalez E., Koposov S. E., Bejar V. J., Lodieu N., Rebolo R., VHS Collaboration, 2013, The Messenger, 154, 35

Meisner A. M., Lang D., Schlegel D. J., 2017, AJ, 153, 38

Milisavljevic D. et al., 2015, ApJ, 815, 120

Minkowski R., 1941, PASP, 53, 224

Modjaz M., Gutiérrez C. P., Arcavi I., 2019, Nat. Astron., 3, 717

Modjaz M., Li W. Chornock R. et al. 2009, AJ, 702, 226

Mokiem M. R. et al., 2007, A\&A, 473, 603

Nicholl M. et al., 2013, Nature, 502, 346

Nicholl M. et al., 2014, MNRAS, 444, 2096

Nicholl M. et al., 2015, MNRAS, 452, 3869

Nicholl M. et al., 2016, ApJ, 826, 39

Nicholl M. et al., 2020, Nat. Astron., 4, 893

Nymark T. K., Fransson C., Kozma C., 2006, A\&A, 449, 171

Ochsenbein F., Bauer P., Marcout J., 2000, A\&AS, 143, 23

Orellana M., Bersten M. C., Moriya T. J., 2018, A\&A, 619, A145

Osterbrock D. E., Ferland G. J., 2006, Astrophysics of Gaseous Nebulae and Active Galactic Nuclei. University Science Books, Sausalito, CA, United States

Pastorello A. et al., 2015, MNRAS, 449, 1941

Pessi P. J. et al., 2019, MNRAS, 488, 4239

Popov D. V., 1993, ApJ, 414, 712

Quimby R. M. et al., 2011, Nature, 474, 487

Quimby R. M. et al., 2018, ApJ, 855, 2

Quimby R. M., Yuan F., Akerlof C., Wheeler J. C., 2013, MNRAS, 431, 912

Reynolds T. M. et al., 2020, MNRAS, 493, 1761

Richmond M. W., 2014, J. Am. Assoc. Var. Star Obs., 42, 333

Roming P. W. A. et al., 2005, Space Sci. Rev., 120, 95

Sánchez-Sáez P. et al., 2019, ApJS, 242, 10

Sanders N. E. et al., 2015, ApJ, 799, 208

Schlafly E. F., Finkbeiner D. P., 2011, ApJ, 737, 103

Schlegel E. M., 1990, MNRAS, 244, 269

Schulze S. et al., 2018, MNRAS, 473, 1258

Schulze S. et al., 2020, preprint (arXiv:2008.05988)

Singh A., Kumar B., Moriya T. J., Anupama G. C., Sahu D. K., Brown P. J., Andrews J. E., Smith N., 2019, ApJ, 882, 68

Smartt S. J. et al., 2015, A\&A, 579, A40

Smith K. W. et al., 2020, PASP, 132, 085002

Smith N., Foley R. J., Filippenko A. V., 2008, ApJ, 680, 568

Soderberg A. M. et al., 2008, Nature, 453, 469 
Soker N., Gilkis A., 2017, ApJ, 851, 95

Speagle J. S., 2020, MNRAS, 493, 3132

Steele I. A. et al., 2004, in Oschmann Jacobus M. J., ed., Proc. SPIE Conf. Ser. Vol. 5489, Ground-based Telescopes. SPIE, Bellingham, p. 679

Sukhbold T., Thompson T. A., 2017, MNRAS, 472, 224

Swann E., Frohmaier C., Nicholl M., Short P., Yaron O., 2019, Transient Name Server Classification Report, 963, 1

Taddia F. et al., 2013, A\&A, 555, A10

Terreran G. et al., 2016, MNRAS, 462, 137

Tody D., 1986, in Crawford D. L., ed., Proc. SPIE Conf. Ser., Vol. 627, Instrumentation in Astronomy VI. SPIE, Bellingham, p. 733

Tonry J. L. et al., 2018, PASP, 130, 064505

Utrobin V. P., 2007, A\&A, 461, 233

Valenti S. et al., 2014, MNRAS, 438, L101

Valenti S. et al., 2016, MNRAS, 459, 3939

van Dokkum P. G., Bloom J., Tewes M., 2012, L.A.Cosmic: Laplacian Cosmic Ray Identification. Astrophysics Source Code Library

Vogl C., Sim S. A., Noebauer U. M., Kerzendorf W. E., Hillebrandt W., 2019, A\&A, 621, A29

Vurm I., Metzger B. D., 2021, preprint (arXiv:2101.05299)

Wheeler J. C., Yi I., Höflich P., Wang L., 2000, ApJ, 537, 810

Woods P. M., Thompson C., 2006, Soft Gamma Repeaters and Anomalous

X-Ray Pulsars: Magnetar Candidates. Cambridge University Press, Cambridge, UK, p. 547

Woosley S. E., Pinto P. A., Hartmann D., 1989, ApJ, 346, 395

Wright A. H. et al., 2016, MNRAS, 460, 765

Wright E. L. et al., 2010, AJ, 140, 1868

Yan L. et al., 2015, ApJ, 814, 108

Yan L. et al., 2017, ApJ, 848, 6

Yaron O., Gal-Yam A., 2012, PASP, 124, 668

Zaritsky D., Kennicutt R. C. Jr, Huchra J. P., 1994, ApJ, 420, 87

\section{APPENDIX A: PHOTOMETRY CODE}

The pipeline of the photometry code which was used to produce the light curve from the LCO and LT photometry is here described. The total flux is calculated from the pre-reduced data (bias, flat) by the Iteratively Subtracted PSF Photometry from PhotUtils (Bradley et al. 2020) - the Point Spread Function (PSF) is taken to be a Gaussian as this is found to produce a good fit and is an acceptable approximation. The PSF fitting is confined to a 50-pixel-width square around the central SN coordinates. With an average FWHM below 10 pixels, this size is assumed to safely include all the associated flux. Alternatives to the Gaussian PSF were also considered - such as an ePSF (effective PSF) constructed from reference stars, as well as aperture photometry. The Gaussian PSF was found to be the method where the scatter between adjacent points was minimized. The magnitude is calculated as below (where $Z P$ is the zero-point):

$\mathrm{Mag}=\mathrm{ZP}-2.5 \log ($ Counts $/$ Exposure $)$.

Valid PSF fits are filtered by setting a threshold of $3 \sigma$ and requiring no close stars which would suggest an unreliable fit. These constraints are optimized through variation and inspection of residuals. The uncertainty is obtained by combining in quadrature the uncertainty in the fit given by the PSF and the uncertainty in the image. The uncertainty in the image is given by

Error $=\sqrt{\frac{\text { Counts }+ \text { Sky }}{\text { Gain }}+\text { Npix } \times(\text { Readnoise }+ \text { Sky })}$,

where Sky is the sky counts over an area the size of the SN, calculated by finding the sigma-clipped mean in the environment surrounding the SN and multiplying by the number of pixels, Npix in the above. Gain and Readnoise come from the header of each fits file. The equation below shows how this uncertainty in counts is
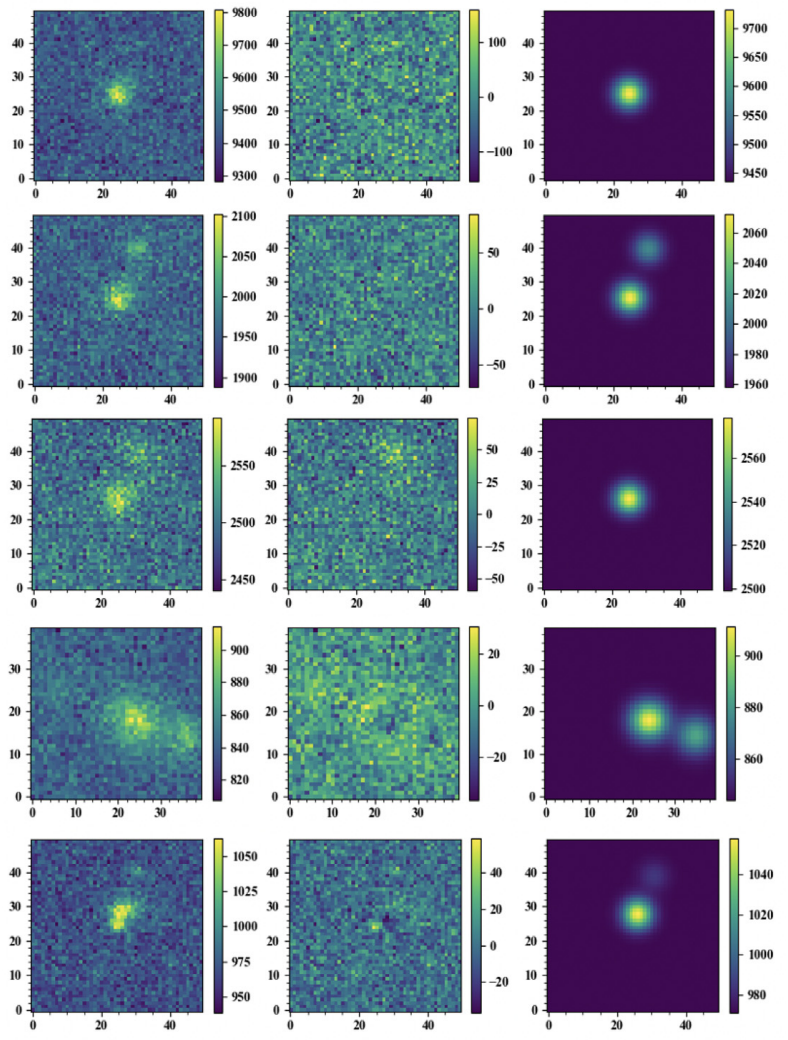

Figure A1. Demonstration of PSF fitting for sample image data. Column 1: Image Data. Column 2: Residual, Column 3: PSF fit. Row 1: $i$-band MJD = 58655, Row 2: $z$-band MJD = 58674, Row 3: $z$-band MJD = 58684, Row 4: Host galaxy in $i$-band, Row 5: $r$-band MJD $=58703$. All images above were taken by the Liverpool Telescope (Steele et al. 2004). The images display the 50-by-50 pixel width square centred on the coordinates of SN 2019hcc, and the colourbar displays the counts as represented by the intensity.

converted to magnitude.

Error $=\frac{2.5}{\ln (10)} \sqrt{\frac{\text { Error(Count })}{\text { Count(Total) }}}$.

This uncertainty is then combined in quadrature with the extinction and the colour uncertainties, which are taken as 0.03 and 0.011 , respectively. These values are taken from Valenti et al. (2016) for one telescope and is carried over as an approximation for the others. Such an assumption might appear unreasonable, but it is indeed acceptable as these terms are a small contribution to the uncertainy budget, and these values are roughly representative (ranges are 0.02-0.09 for extinction, and $0.011-0.036$ for the colour). Cosmic ray artifacts are removed using LACOSMIC (van Dokkum, Bloom \& Tewes 2012).

The $Z P$ are found by fitting the PSF to reference stars and reversing the magnitude calculation. This is achieved in the code by accessing the Pan-STARRS (Chambers et al. 2016) catalogue using Vizier (Ochsenbein et al. 2000) and selecting all available stars in a 5 arcmin radius around the $\mathrm{SN}$ coordinates (see Fig. 1). To improve the quality of the PSF fit, multiple images taken on the same night (when available) were (and can be in a general workflow) aligned and stacked using the SNOoPY (SuperNOva PhotometrY) package. ${ }^{6}$

${ }^{6} \mathrm{SNOoPy}$ is a package for SN photometry using PSF fitting and/or template subtraction developped by E. Cappellaro. A package description can be found at http://sngroup.oapd.inaf.it/ecsnoopy.html. 
Template subtraction for this code is as follows: host images (which could be combined using SNOoPY, excluding poorer images) and the flux of both the host image and each SN image are found using the PSF fitting method described above. equation (A1) is used to convert the host flux to what it would be if it had the same $Z P$ and exposure of the SN image, then the fluxes are subtracted, and the uncertainties propagated.

Fig. A1 displays the PSF fitting for a few example images. The first column displays the image data, whilst the second and third show the residual and PSF fit, respectively. As can be seen, the Gaussian PSF fit can produce relatively clean residual images, and the code recognizes multiple sources.

\section{APPENDIX B : DATA}

The spectroscopy log of SN 2019hcc observations is reported in Table B1, while the host galaxy magnitudes are in Table B2 and the supernova photometry in Tables B3-B6.

Table B1. Spectroscopy data as displayed in Fig. 9. The resolutions of the spectra are found from measuring the skylines using IRAF, excluding the SOAR spectrum resolution which was was taken from http://www.ctio.noao. edu/soar/content/goodman-spectrograph-gratings.

\begin{tabular}{cccccc}
\hline Epoch & $\begin{array}{c}\text { Phase from } \\
\text { maximum (d) }\end{array}$ & Instrument & Grisms & Range $(\AA)$ Resolution $(\AA)$ \\
\hline 58643 & 7 & EFOSC2 & Gr 11 & $3380-7520$ & 13.7 \\
58655 & 19 & SOAR & $400 \mathrm{~mm}$ & $3200-8500$ & 6.0 \\
58665 & 29 & EFOSC2 & Gr 13 & $3685-9315$ & 25.7 \\
58689 & 53 & EFOSC2 & Gr 13 & $3685-9315$ & 17.4 \\
58717 & 81 & EFOSC2 & Gr 13 & $3685-9315$ & 17.1 \\
58814 & 178 & EFOSC2 & Gr 13 & $3685-9315$ & 17.3 \\
59149 & Host spectrum & EFOSC2 & Gr 13 & $3685-9315$ & 16.0 \\
\hline
\end{tabular}

Table B2. The measured apparent magnitudes of the host galaxy for SN 2019hcc from LT and LCO images.

\begin{tabular}{lc}
\hline Filter & Apparent magnitude \\
\hline$B$ & $20.70(0.18)$ \\
$V$ & $20.05(0.23)$ \\
$g$ & $20.68(0.24)$ \\
$r$ & $20.67(0.28)$ \\
$i$ & $20.37(0.29)$ \\
$z$ & $20.56(0.18)$ \\
\hline
\end{tabular}

Table B3. NIR GROND magnitudes as seen in Fig. 5.

\begin{tabular}{lcccc}
\hline MJD & $\begin{array}{c}\text { Phase from } \\
\text { maximum }(\mathrm{d})\end{array}$ & $J$ & $H$ & $K$ \\
\hline 58644 & 8 & $18.08(0.17)$ & $17.87(0.25)$ & $17.64(0.50)$ \\
58648 & 12 & $18.08(0.17)$ & $17.90(0.25)$ & $17.80(0.03)$ \\
58661 & 25 & $18.23(0.18)$ & $17.64(0.23)$ & $17.12(0.28)$ \\
58667 & 31 & $18.26(0.18)$ & $17.91(0.23)$ & $17.56(0.32)$ \\
58674 & 38 & $18.33(0.17)$ & $18.00(0.23)$ & - \\
\hline
\end{tabular}


Table B4. Photometry data shown in Fig. 5.

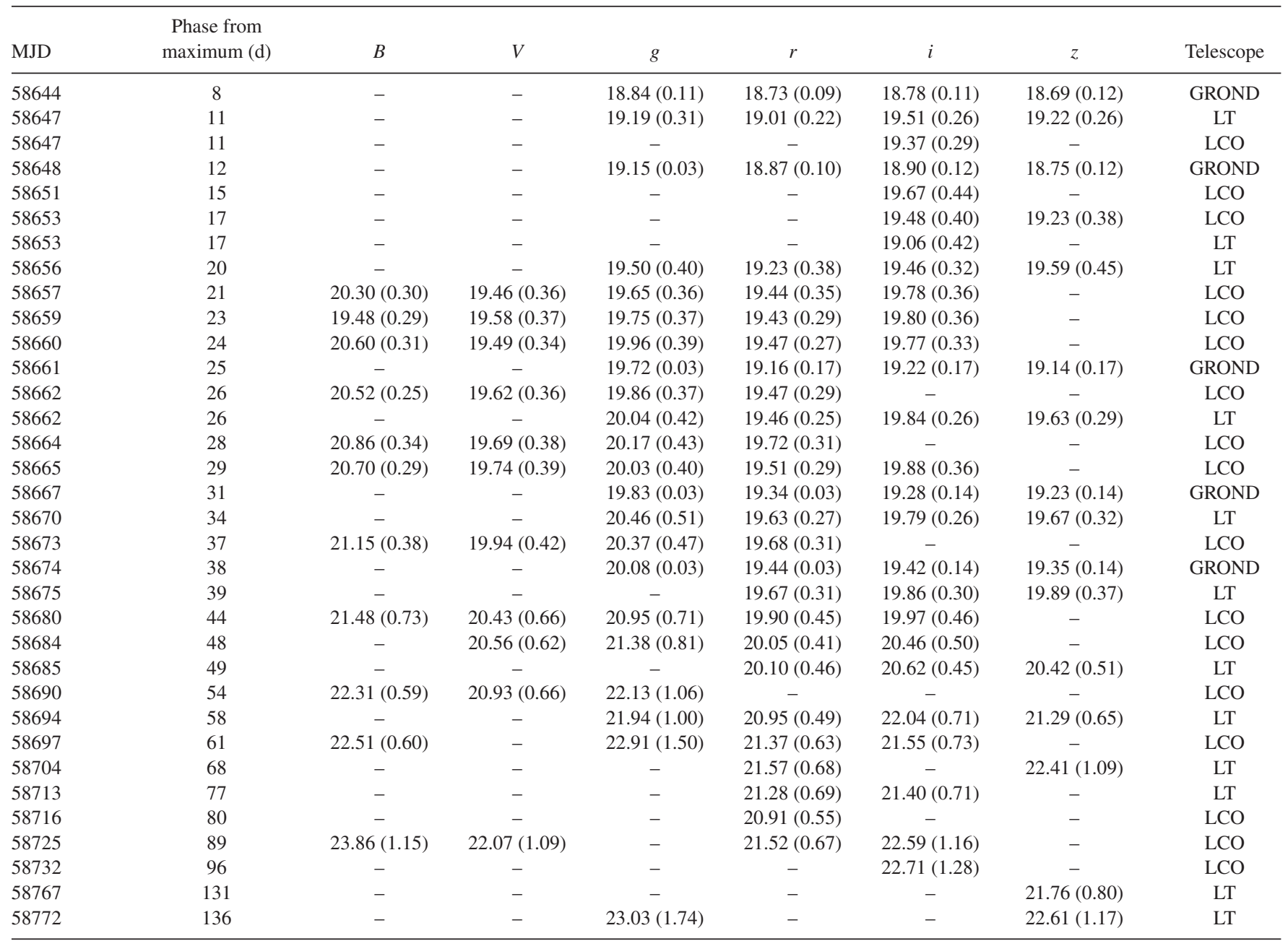

Table B5. Swift AB magnitudes as seen in Fig. 5.

\begin{tabular}{lccccc}
\hline MJD & $\begin{array}{c}\text { Phase from } \\
\text { maximum }(\mathrm{d})\end{array}$ & UVM2 & UVW1 & UVW2 & $u$ \\
\hline 58645 & 9 & $20.72(0.14)$ & $20.21(0.24)$ & $20.71(0.20)$ & $19.68(0.21)$ \\
58651 & 15 & $20.78(0.31)$ & $>20.39$ & $20.72(0.31)$ & $>19.72$ \\
58658 & 22 & $20.80(0.15)$ & $20.50(0.20)$ & $21.17(0.21)$ & $20.54(0.32)$ \\
58660 & 24 & $21.30(0.23)$ & $20.67(0.24)$ & $21.72(0.32)$ & $>20.58$ \\
58663 & 27 & $20.76(0.16)$ & $20.79(0.24)$ & $20.95(0.19)$ & $>20.70$ \\
58666 & 30 & $21.32(0.21)$ & $20.67(0.22)$ & $21.14(0.21)$ & $>20.71$ \\
\hline
\end{tabular}


Table B6. ATLAS AB magnitudes as reported in Fig. 5.

\begin{tabular}{|c|c|c|c|c|c|c|c|}
\hline MJD & $\begin{array}{l}\text { Phase from } \\
\text { maximum (d) }\end{array}$ & Cyan & Orange & MJD & $\begin{array}{l}\text { Phase from } \\
\text { maximum (d) }\end{array}$ & Cyan & Orange \\
\hline 58609 & -27 & $>20.61$ & - & 58643 & 7 & - & $18.87(0.15)$ \\
\hline 58609 & -27 & $>20.43$ & - & 58643 & 7 & - & $18.96(0.17)$ \\
\hline 58609 & -27 & $>19.91$ & - & 58645 & 9 & - & $18.80(0.13)$ \\
\hline 58611 & -25 & - & $>20.15$ & 58645 & 9 & - & $18.69(0.11)$ \\
\hline 58617 & -19 & - & $>20.06$ & 58647 & 11 & - & $19.16(0.21)$ \\
\hline 58617 & -19 & - & $>20.12$ & 58647 & 11 & - & $19.16(0.21)$ \\
\hline 58617 & -19 & - & $>20.20$ & 58649 & 13 & - & $18.90(0.31)$ \\
\hline 58617 & -19 & - & $>20.44$ & 58649 & 13 & - & $18.81(0.26)$ \\
\hline 58619 & -17 & - & $>19.71$ & 58659 & 23 & - & $19.01(0.21)$ \\
\hline 58619 & -17 & - & $>19.65$ & 58659 & 23 & - & $19.54(0.33)$ \\
\hline 58620 & -16 & - & $>19.13$ & 58665 & 29 & $20.07(0.29)$ & - \\
\hline 58620 & -16 & - & $>19.22$ & 58665 & 29 & $19.91(0.26)$ & - \\
\hline 58620 & -16 & - & $>19.40$ & 58665 & 29 & $19.57(0.21)$ & - \\
\hline 58621 & -15 & - & $>19.41$ & 58667 & 31 & - & $19.62(0.27)$ \\
\hline 58621 & -15 & - & $>19.51$ & 58667 & 31 & - & $19.70(0.27)$ \\
\hline 58621 & -15 & - & $>19.56$ & 58667 & 31 & - & $19.17(0.16)$ \\
\hline 58621 & -15 & - & $>19.60$ & 58667 & 31 & - & $19.31(0.21)$ \\
\hline 58623 & -13 & - & $>17.86$ & 58669 & 33 & $19.89(0.31)$ & - \\
\hline 58623 & -13 & - & $>19.14$ & 58669 & 33 & $19.55(0.24)$ & - \\
\hline 58623 & -13 & - & $>18.98$ & 58669 & 33 & $19.17(0.17)$ & - \\
\hline 58623 & -13 & - & $>19.20$ & 58670 & 34 & $19.99(0.31)$ & - \\
\hline 58631 & -5 & - & $19.25(0.22)$ & 58670 & 34 & $20.16(0.34)$ & - \\
\hline 58631 & -5 & - & $18.97(0.24)$ & 58671 & 35 & $19.92(0.30)$ & - \\
\hline 58637 & 1 & $18.54(0.09)$ & - & \multirow{2}{*}{\multicolumn{4}{|c|}{ This paper has been typeset from a $\mathrm{T}_{\mathrm{E}} \mathrm{X} / \mathrm{L} \mathrm{A} \mathrm{T}_{\mathrm{E}} \mathrm{X}$ file prepared by the author. }} \\
\hline 58643 & 7 & - & $18.90(0.15)$ & & & & \\
\hline
\end{tabular}

Table B6 - continued 